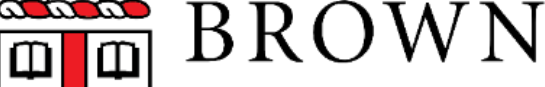 \\ (由) Orlando Bravo Center \\ for Economic Research
}

\section{Financial Aid and Early Admissions at Selective Need-Blind Colleges}

\author{
Bravo Working Paper \# 2020-024 \\ Zeky Murra-Anton \\ Brown University
}

\begin{abstract}
I study a college-admissions model with two need-blind colleges and heterogeneous students. In a game in which colleges can choose a financial aid policy and either binding, nonbinding, or no early admissions, a unique equilibrium outcome exists. In equilibrium - and consistent with data-the more prestigious and wealthier college is more selective, has a more generous financial aid policy, and offers nonbinding early admissions while the other college offers a binding program. Compared to the counterfactual in which only regular admissions are offered, early admissions make the more prestigious college worse off but make all students and the other college better off.
\end{abstract}

Keywords: college early admissions, need-blind admissions, financial aid, decentralized matching JEL Codes: $\quad$ D47, I23, L31.

*Phone: +1 401-863-3836, E-mail: zeky.murra@brown.edu. This paper supersedes the one titled "College Early Admissions: Determinants and Welfare." I thank Roberto Serrano, Jesse Shapiro, Neil Thakral, Jack Fanning, Bobby Pakzad-Hurson and Lorenzo Aldeco for their useful feedback. Declarations of interest: none. 


\title{
Financial Aid and Early Admissions at Selective Need-Blind Colleges
}

\author{
Zeky Murra-Anton \\ Brown University* \\ Date: February 12, 2021
}

\begin{abstract}
I study a college-admissions model with two need-blind colleges and heterogeneous students. In a game in which colleges can choose a financial aid policy and either binding, nonbinding, or no early admissions, a unique equilibrium outcome exists. In equilibrium—and consistent with data-the more prestigious and wealthier college is more selective, has a more generous financial aid policy, and offers nonbinding early admissions whereas the other college offers a binding program. Compared to the counterfactual in which only regular admissions are offered, early admissions make the more prestigious college worse off but all students and the other college better off.
\end{abstract}

Keywords: college early admissions, need-blind admissions, financial aid, decentralized matching

JEL Codes: D47, I23, L31.

*Phone: +1 401-863-3836, E-mail: zeky.murra@brown.edu. This paper supersedes the one titled "College Early Admissions: Determinants and Welfare." I thank Roberto Serrano, Jesse Shapiro, Neil Thakral, Jack Fanning, Bobby Pakzad-Hurson and Lorenzo Aldeco for their useful feedback. Declarations of interest: none. 
Among the top colleges in the US, all but two offer early admissions-they allow students to apply and receive a formal decision prior to the regular admissions process-and about 86 percent reports to use a need-blind policy — they claim to admit students based only on academic merit and commit to covering a significant part of students' financial need with aid. Beyond these similarities, however, college admissions at top schools are far from homogeneous. On the one hand, colleges use different early admissions formats, with early decisions (ED) and restrictive early action (REA) being the most common. ${ }^{1}$ On the other hand, the facts that colleges have diverse endowments and that need-blind admissions are costly and limit schools' control over their financial commitments ${ }^{2}$ lead to a wide spectrum of financial-aid policies.

How do need-blind colleges choose an early-admissions program? How do they determine their financial aid policy? What are the welfare implications of early and need-blind admissions at top schools? These questions remain open and I show that their answer is tightly related. Two observations hint that early admissions might play a key role in helping need-blind schools manage their financial commitment: first, that among colleges using early admissions, all but the wealthiest-Harvard, Stanford, Princeton, and Yale — use ED. Second, that low-income students avoid ED because it reduces their chances to compare financial aid offers (Antecol and Kiholm Smith, 2012; Avery et al., 2009).

To study the relationship between early admissions, need-blind policies and financial aid, I construct a model with two need-blind colleges and three novel features: colleges choose a financial aid policy, they also choose which early-admissions program to offer (if any), and students are heterogeneous in their wealth. In my model, students have preferences for financial aid and taste, which differ among high- and low-income students, whereas colleges have preferences for student quality and expenditure on financial aid. Colleges' only difference is the weight that financial aid considerations bear in the admissions process.

My analysis relies on two reasonable assumptions: first, that low-income students strongly benefit from comparing financial aid offers, and second, that the more prestigious college is wealthier and puts less weight on financial considerations. Using a refined version of perfect Bayesian equilibrium, I analyze a game with three stages. In the first one, colleges simultaneously decide on a financial aid policy. In the second one, colleges simultaneously decide which early-admissions program to offer, if any. In the third stage, students decide where to apply-early and regular-and

${ }^{1}$ The main difference is that upon admission, an ED applicant is committed to enrolling, whereas an REA applicant is not. ${ }^{2}$ Only 55 percent of the enrolled students at the top private selective colleges paid full price in the first decade of the twenty-first century (Hill and Winston, 2006). 
colleges decide which students to admit.

Need-blind admissions create idiosyncratic uncertainty: colleges only observe students' quality at the time of the application, whereas demonstration of financial need is a noisy, case-by-case process. ${ }^{3}$ Considering financial aid allows me to highlight the important trade-off that colleges face between student quality and financial aid expenditure, which is key for my results. In some cases, this trade-off can be strong enough to lead to justified envy: a college rejects a relatively high-quality student in favor of a lower-quality one, just to save on financial aid.

My first main result, Theorem 1, shows that there is a unique equilibrium outcome in which the wealthier and more prestigious school (college A) offers REA (the other college, college B, offers ED), is more selective, and institutes a more generous financial aid policy than college B. Moreover, both colleges favor early applicants, and low-income students avoid the ED binding commitment. These findings are consistent with empirical facts.

A competitive force drives my result, and it rests on two observations. First, ED turns the early-admissions process into a wealth-screening device for college B (the less wealthy one): when low-income students benefit from comparing financial aid offers, they avoid ED's binding commitment. Second, by favoring early applicants, college B attracts and secures some high-income, high-quality students with a preference for college A that value a higher overall chance of admission over fit. In this way, college B has enrollment control over a group of high-quality, high-income students that allows it to reduce the financial commitments associated with the admissions process. Meanwhile, college A prefers using REA to the alternatives: if it does not offer early admissions, some of the students with a weak preference for it enroll through college B's ED out of impatience, whereas if it offers ED, it deters low-income students from applying early.

My second main result, Theorem 2, compares the early-admissions equilibrium with a counterfactual in which, all else equal, colleges are only allowed to offer regular admissions and students apply accordingly. I show that when college B is willing to enroll through its regular admissions process the top students rejected by college A's early program, under early admissions college B and all students are better off whereas college A is worse off. The case of low-income students is particularly interesting as it goes against the popular belief that they are hurt by early admissions (Avery et al., 2009; Chapman and Dickert-Conlin, 2012; Fetter, 1997). The result follows from the fact that when colleges offer early admissions, competition leads the more selective and prestigious college to weaken its admission standards, maximizing the low-income students' chance to be admitted to both colleges and compare financial aid offers.

${ }^{3}$ It is not until after an admission decision that the college and student learn the exact financial aid offer. 
An important caveat is that my model is tailored to the top private selective colleges because it has embedded institutional features that are hard to find in other institutions. My goal is to address questions about financial aid and early admissions at top private schools, which enroll close to 40,000 students every year and are constant objects of economic policy. For this reason, the reader must be careful when applying my results to other schools. The main issues that must be addressed before such an extrapolation can be successful relate to the wealth of the schools-for example, whether colleges can afford to be need-blind and flexible in their expenditure on financial aid.

The rest of the article is organized as follows. In Section 1, I summarize the related literature and the key institutional features of the market and I present stylized facts. In Section 2, I present the model. In Section 3, I present my equilibrium results. In Section 4, I present my welfare results'. In Section 5 I present extensions to my model, such as general students' preferences, general colleges' preferences and justified envy. Finally, in Section 6, I conclude.

\section{Background}

\subsection{Related Literature and Contributions}

My first contribution is to explain the real-world early-admissions market configuration as a result of college competition. This cannot be deduced from related articles, such as Avery and Levin (2010), Kim (2010), and Lee (2009), because they abstract from key competitive aspects of the market. My model is closest to that in Avery and Levin (2010), with two main differences: I allow need-blind colleges to choose an early-admissions program and a financial aid policy, and I consider students heterogeneous in wealth. The goal of Avery and Levin (2010) is to analyze how colleges with a preference for student fit use early admissions to improve their matches and yield rates. ${ }^{4}$

Kim (2010) studies the relationship between ED and students' wealth. That paper considers need-blind, tuition-maximizing colleges that use ED to identify wealthy students. Unlike my model, Kim (2010)'s only considers ED (not REA) and does not allow colleges to decide whether to offer early admissions. Meanwhile, Lee (2009) focuses on how privately informed employers use early contracting to avoid the winner's curse that hiring a worker might bring. The paper exemplifies its findings with early admissions. Although their framework allows colleges to not offer early admissions, it abstracts from two factors that are central in my analysis: financial aid and colleges' option to use REA programs.

${ }^{4}$ The yield rate is defined as the proportion of admitted students that enroll. 
My second contribution is to study the market welfare after accounting for college competition through endogenously-chosen early admissions programs and financial aid policies. My predictions significantly depart from those that can be made based on Avery and Levin (2010), Kim (2010), and Lee (2009). For instance, Avery and Levin (2010) finds that every college is made better off by REA, Lee (2009) finds that every college is made better off by early admissions, and Kim (2010) finds that ED does not make a budget-constrained college better off. My model, in contrast, predicts that among colleges, only those using ED are made better off by early admissions.

My third contribution, a theoretical one, is to highlight the trade-off between student quality and financial aid that colleges face, which can lead to justified envy. The analysis of this trade-off is absent from other college admission models that do not consider financial aid, such as Avery and Levin (2010), Lee (2009), Chade et al. (2014), as well as from other higher-education related work considering financial aid, such as Epple and Romano (1998), Epple et al. (2006), and Kim (2010).

\subsection{Institutional Features}

I present a brief summary of key institutional features of the market. Avery et al. (2009) and Ashby (2006) are good sources for a detailed treatment of the institutional features of early and need-blind admissions, respectively.

Early Admissions: Among top colleges, early-admissions programs mainly take the form of ED or REA. In both, a student is allowed to apply early to only one college and receives an admission decision prior to the start of the regular admissions process. The student cannot reapply to the college she applied to early. If she gets rejected early, she can proceed with the regular admission process at other colleges. If the student is admitted to an REA program, she can enroll at any point before the regular admission process concludes and can apply to other schools through their regular admissions processes. If the student is admitted to an ED program, she must enroll and she commits to withdrawing any regular admissions applications.

Need-Blind Admissions and Financial Aid: Colleges commit to considering only merit, not financial need, in granting admission. Often, colleges have separate applications for admissions and financial aid, which are evaluated independently. The college, prior to the application process, announces a general financial aid policy. In it, the college specifies general criteria defining financial need and the proportion of it to be covered with financial aid upon enrollment. In practice, demonstrated need is privately determined on a case-by-case basis by the financial office. Multiple idiosyncratic factors play a role, such as the evidence that students provide and the judgment of the evaluator when applying the determination criteria (Avery et al., 2009), which often lead to different 
evaluations of the same candidate at different schools.

\section{Model}

There is a unit measure continuum of students and two need-blind colleges, A and B, and two admission processes, early and regular. I define $\mathcal{S}=[0,1]$ to be the set of students. I denote a given college by $j \in\{A, B\}$, and by $-j$ I denote the college that is not $j$. Likewise, I denote a given student by $i \in \mathcal{S}$, and by $-i$ I denote the students that are not $i$.

\subsection{Students}

Each student is characterized by a triple $(q, y, n) \in[0,1] \times[\underline{y}, \bar{y}] \times\{\bar{n}, \underline{n}\} \equiv \mathcal{T}$, where $q$ represents the quality of her application, $y$ represents her preference or taste for colleges, and $n$ represents her financial need. I let $0 \leq \underline{n}<\bar{n}$, so a student with financial need $\bar{n}$ can be thought of as low-income, whereas one with financial need $\underline{n}$ can be thought of as high-income. I also let $-\infty<\underline{y}<0<\bar{y}<\infty$; students with taste $y>0$ prefer college A over college $\mathrm{B}$, whereas the opposite is true for students with taste $y<0$.

\subsubsection{Student Characteristics}

Student characteristics $(q, y, n)$ are distributed according to the common-knowledge cumulative distribution function $G$, which is absolutely continuous in $(q, y)$ for every $n$ and is also full-support. I let $G_{z}$ denote $z$ 's marginal cumulative distribution, for $z \in\{q, y, n\}$. Student characteristics are independent among students. To induce a ranking among schools, I assume that students prefer college A over college B on average-that is, $G_{y}(0 \mid q, n) \in\left(0, \frac{1}{2}\right)$ for every $q$ and $n$. I interpret this to mean that college $\mathrm{A}$ is more prestigious than college $\mathrm{B}$.

In line with the evidence from Avery et al. (2009), I let high-income students have a greater chance of being high-quality and having a taste for the more prestigious college. Formally, I assume that $(q, y,-n)$ are positively affiliated. ${ }^{5}$ Finally, I assume that students do not directly observe their quality, only their taste and financial need. This does not mean, however, that students are

${ }^{5}$ Let $g\left(q, y \mid n=n^{\prime}\right)$ be the joint density of $(q, y)$ conditional on $n=n^{\prime}$ and-with a slight abuse of notation$g\left(q, y, n^{\prime}\right) \equiv g\left(q, y \mid n=n^{\prime}\right) \mathbb{P}\left(n=n^{\prime}\right)$ the joint "density" of $\left(q, y, n^{\prime}\right)$. Then, for any $t=(q, y, n)$ and $t^{\prime}=$ $\left(q^{\prime}, y^{\prime}, n^{\prime}\right)$, I assume that $g\left(t \wedge t^{\prime}\right) g\left(t \vee t^{\prime}\right) \geq g(t) q\left(t^{\prime}\right)$, with $t \wedge t^{\prime} \equiv\left(\max \left\{q, q^{\prime}\right\}, \max \left\{y, y^{\prime}\right\}, \min \left\{n, n^{\prime}\right\}\right)$ and $t \vee t^{\prime} \equiv\left(\min \left\{q, q^{\prime}\right\}, \min \left\{y, y^{\prime}\right\}, \max \left\{n, n^{\prime}\right\}\right)$. 
completely uninformed about the quality of their application; the affiliation of $(q, y, n)$ implies that students can make inference about $q$ by observing $(y, n)$. In that sense, $(y, n)$ serves the double purpose of being a signal of the student's true quality, as well as her characteristics. ${ }^{6}$

\subsubsection{Students' Preferences}

Low-income students are more sensitive to financial aid than high-income ones. For ease of exposition, I assume that students' preferences are lexicographic in financial aid and taste: low-income students' first preference criterion is financial aid and consider taste only to break ties, whereas the opposite is true for high-income students. As in any model with absolutely-continuous distributions, ties only happen with probability zero, so I proceed as if low-income students' preferences were entirely determined by financial aid and high-income students', by taste; in Section 5, I show that my results extend to the case where students have general preferences depending on both, taste and financial aid.

Formally, if a low-income student enrolls at college $j$ with a financial aid offer of $x_{j}$, she gets utility of $v_{l}\left(x_{j}\right)>0$, where $v_{l}$ is a strictly increasing function. Among high-income students, those with $y>0$ prefer college A, whereas the ones with $y<0$ prefer college B. A high-income student with taste $y$ that enrolls at college $j$ gets a utility of $v_{h}(j, y)>0$, where $v_{h}(A, y)$ and $v_{h}(B, y)$ are strictly positive and continuous in $y, v_{h}(A, y)$ is strictly increasing in $y$, and $v_{h}(B, y)$ is strictly decreasing in $y$. The student with $y=0$ is indifferent between the two colleges, so $v_{h}(A, 0)=v_{h}(B, 0)$. I assume that an extreme taste for a given college translates into a very strong preference - that is, $\lim _{y \rightarrow \bar{y}} v_{h}(A, y)=\lim _{y \rightarrow \underline{y}} v_{h}(B, y)=\infty$. I normalize the utility of not enrolling at any college to zero for every student. Students, all else equal, prefer to enroll in early admissions: one of the main documented reasons why students apply early is that being admitted early reduces the stress and length of the admissions process (Avery et al., 2009). To model this feature, I let students discount regular enrollment by a factor of $\delta \in(0,1)$.

A key assumption of my model is that low-income students put significant weight on being able to compare financial aid offers, namely, that they prefer to enroll regular with the best of two financial aid offers than enrolling early with only one. Mathematically, this assumption is that $\bar{v}\left(\rho_{B}\right)>\frac{\bar{v}_{A}\left(\rho_{B}\right)}{\delta G^{*}}$ for every $\rho_{B}$, where $\rho_{j}$ is college j's financial aid policy (formalized in the next section), $\bar{v}\left(\rho_{B}\right)$ is a low-income student's expected utility when she receives two offers, $\underline{v}_{j}\left(\rho_{B}\right)$ is her

${ }^{6} \mathrm{~A}$ more complicated framework is one in which students observe also a monotonic signal $\omega$ of their quality. However, by properly re-defining the support and distribution of $(q, y, n)$, the posterior belief of $q$ from observing $(n, y)$ can be made equivalent to the posterior belief of $q$ from observing $(n, y, \omega)$. 
expected utility when she only receives an offer from college $j$, and $G^{*}$ is her smallest probability of admission at a given college that exhausts capacity. ${ }^{7}$ This assumption guarantees that low-income students never value enrolling early over comparing financial aid offers, and that the cost associated with applying ED—having to enroll without comparing offers if admitted—is significant.

\subsection{Colleges}

\subsubsection{College Characteristics}

In my model, each college has fixed capacity $\kappa<\frac{1}{2}$ and both schools are need-blind, in the sense that they make admission decisions only based on students' quality. Throughout the admissions process, each college puts a weight $\alpha_{j}$ on financial aid considerations; I assume that this weight is enough to reflect the college's budget situation so, after accounting for $\alpha_{j}$, the college only focuses on managing its capacity. Although I remain agnostic to how $\alpha_{j}$ is determined, one intuitive interpretation is that $\alpha_{j}$ is the college's sensitivity to its expenditure in financial aid, which is decreasing function of its endowment. Letting $x_{j}$ be the financial aid offer that college $j$ makes to a given student, the college's ex post utility from enrolling her when she has quality $q$ is $u(q)-\alpha_{j} x_{j}$, for $u_{j}(q)>0$, a strictly increasing, concave and differentiable function. ${ }^{8}$

For a given $q$, college $j$ 's marginal rate of substitution implies that its willingness to sacrifice student quality to save on financial aid (WTS) is $\frac{\alpha_{j}}{u^{\prime}(q)}$. In particular, for any $q$, college A's WTS, relative to college B's, is $\frac{\alpha_{A}}{\alpha_{B}}$. The only difference between colleges in my model is their WTS. To capture the notion that college A (the more prestigious among students) is the wealthier one, I assume that its WTS is smaller than college B's-that is, $0=\alpha_{A}<\alpha_{B}$. Letting $\alpha_{A}=0$ is a simplifying assumption; in Section 5, I show that my results extend for a small $0<\alpha_{A}$.

I make two additional assumptions about colleges. First, I assume that $\alpha_{B}<\frac{u(0)}{\bar{n}}$. Keeping $\alpha_{j}$ bounded from above by $\frac{u(0)}{\bar{n}}$ guarantees that colleges see all students - independently of their financial need - as worth enrolling if capacity is available. Second, I assume that colleges break ties in favor of the strategy profile that leads to the most low-income early applicants. I base this assumption on the fact that many of the early-admissions format changes in the 2000s were made to increase the number of low-income early applicants.

${ }^{7}$ Formally, $\bar{v}\left(\rho_{B}\right)=\mathbb{E}\left(\max \left\{v_{l}\left(x_{A}\right), v_{l}\left(x_{B}\right)\right\} \mid n=\bar{n}, \rho_{A}=1, \rho_{B}\right), \underline{v}_{j}\left(\rho_{B}\right)=\mathbb{E}\left(v_{l}\left(x_{j}\right) \mid n=\bar{n}, \rho_{A}=1, \rho_{B}\right)$ and $G^{*}=1-\sup _{y} G_{q}(\kappa \mid n=\bar{n}, y)$. I focus on $\rho_{A}=1$ as it is college A's dominant strategy (see Theorem 1).

${ }^{8}$ This model specification is equivalent, as Appendix A.1 shows, to one in which $\alpha_{j}$ is a penalty (reward) to the college for exceeding (staying under) a financial aid budget, and the college's preferences depend only on $q$. 


\subsubsection{Financial Aid}

A central aspect of financial aid in real life is that it generates uncertainty for admissions offices and students at the moment of the application: colleges do not observe the students' financial need until after admitting them (need-blind admissions), and neither colleges nor students know exactly how much of the financial need will be satisfactorily demonstrated, as the determination process is case-by-case and noisy.

To model this uncertainty, I assume that the admissions office of a college is independent of its financial office. The former chooses and applies an admission policy, whereas the latter evaluates the financial need of the admitted students and extends a financial aid offer. Formally, at the admissions process, a student and the admissions office expect the financial office to extend an offer of $x_{j}=\rho_{j} \hat{n}_{j}$ upon admission, where $\hat{n}_{j} \sim F(\cdot \mid n)$ is the student's demonstrated level of need, $F$ is a common-knowledge continuous uniform distribution with support on $\left[0, b_{n}\right]$ and mean $n$, for some constant $b_{n}$, and $\rho_{j} \in[0,1]$ is the the proportion of demonstrated need that the college covers with aid.

During the admissions process, the financial aid policy $\rho_{j}$ is taken as given; however, it is determined strategically by the college at an earlier stage (more detail is provided in the following section). Implicit in my framework is the assumption that both colleges have the same ability to determine financial need: for a given student, both colleges draw from the same distribution $F$. I further assume that the difference in colleges' financial-need evaluation is purely idiosyncratic: conditional on $n$, a given student's draws $\hat{n}_{A}$ and $\hat{n}_{B}$ are independent, as are the draws across students.

In principle, the financial aid office could be strategic; nonetheless, in equilibrium, students and the admissions office would take its optimal behavior as given. Therefore, I focus on the admissions office and take the financial office to be summarized by the distribution $F$. Under this approach, colleges' beliefs account for the fact that students might not be able to demonstrate their full need: during the admissions process, colleges consider the financial need of their admitted students to be a random draw from $F$. Moving forward, by "the college," I mean "the admissions office." 


\subsection{The Early-Admissions Game, Strategies, and Solution Concept}

\subsubsection{The Early Admissions Game and Strategies}

I assume that a student plans to apply through the regular admissions process (going forward, "regular") to every college she is allowed to. ${ }^{9}$ This simplifying assumption allows me to summarize the students' strategy through their early choice without affecting the equilibrium outcome of the game. The game has three stages:

1. Colleges simultaneously choose a financial aid policy $\rho_{j}$ and commit to it by making it public.

2. Colleges simultaneously choose an early-admissions program $E_{j} \in\{E D, R E A, \emptyset\}$ and commit to the choice by making it public.

3. At the third stage, students simultaneously designate a feasible early application $a_{i} \in\{A, B, \emptyset\}$ and colleges simultaneously determine which students are admitted early and regular.

In the early admissions game, colleges are allowed to not offer early admissions $\left(E_{j}=\emptyset\right)$ and students are allowed to not apply early $\left(a_{i}=\emptyset\right)$. I focus on symmetric pure strategies in the sense that all the students with the same characteristics behave the same. Denote by $p \in\{E, R\}$ the early and regular application processes. An admission policy $\sigma_{j}$ is the function that specifies the proportion of applicants with each quality level $q \in[0,1]$ to be admitted in each application process. Formally, $\sigma_{j}:\{E, R\} \times[0,1] \rightarrow[0,1]$. This definition allows the admission policy to differ for early and regular applicants of a given college. The admission policy for process $p$ is denoted by $\sigma_{j}^{p} \equiv \sigma_{j}(p, \cdot)$. When $\sigma_{j}^{p}(q) \in(0,1)$, only a fraction of the applicants with quality $q$ are admitted; I assume that they are chosen uniformly at random.

The application strategy for student $i$ is summarized by her early-admissions choice $a_{i}$. A strategy for a college is a financial aid policy, an early-admissions program to offer, and an admission policy, collectively denoted by $\Sigma^{j}=\left(\rho_{j}, E_{j}, \sigma_{j}\right)$. By $\mu$ I denote the system of beliefs that colleges hold about their applicants' unknown characteristics. Letting $a=\left(a_{i}\right)_{i \in \mathcal{S}}$, I refer to $\Sigma=\left(\Sigma^{A}, \Sigma^{B}, a, \mu\right)$ as a strategy profile. Going forward, it is useful to define $\Sigma_{k}$ as $\Sigma$ without its element $k$. For instance, $\Sigma_{E_{A}}=\left(\left(\rho_{A}, \sigma_{A}\right), \Sigma^{B}, a, \mu\right)$.

\subsubsection{Solution Concept}

To analyze the game, I use a refined version of perfect Bayesian equilibrium (PBE). Definition 1 formalizes a PBE for the early admissions game and Definition 2, its refinement.

${ }^{9}$ Students admitted ED to a college cannot apply regular anywhere else, whereas students rejected early from a college cannot reapply. 
Definition 1. A PBE is a strategy profile $\Sigma$ such that

(i) for each college $j$, given $\Sigma_{\rho_{j}}, \rho_{j}$ is an optimal financial aid policy;

(ii) for each college $j$, given $\Sigma_{E_{j}}$, it is optimal to offer the early-admissions program $E_{j}$;

(iii) for each college $j$, given $\Sigma_{\sigma_{j}}, \sigma_{j}$ is an optimal admission policy;

(iv) for each student $i$, given $\Sigma_{a_{i}}, a_{i}$ is an optimal application strategy; and

(v) the beliefs $\mu$ are computed with Bayes' rule for each information set with strictly positive probability under $\Sigma_{\mu}$.

Definition 2. An early-admissions equilibrium (EA equilibrium) is a PBE that satisfies the following refinement: if a college offers an early-admissions program, there is a strictly positive measure set of students that apply to it, are admitted, and enroll. Furthermore, no college achieves an admission outcome by threatening to not admit any regular applicant.

In college-admissions models, equilibria in which colleges do not admit any student or no student applies to a college often arise; my model is not an exception and, in some scenarios, colleges might try to sustain an outcome by offering an early admissions program but not admitting any student to it. These outcomes are inconsistent with data, however: when a college offers early admissions, its enrolled class comprises significant proportions of early and regular applicants, as Appendix B shows. The refinement in Definition 2 rules out such possible PBEs.

A final remark is that there is no need for off-equilibrium-path beliefs, as there is no uncertainty between stages: colleges make public the financial aid policy after the first stage and the earlyadmissions market configuration after the second stage. Therefore, in the event that an offequilibrium-path information set is reached, colleges and students can observe the deviation from the financial aid policy or early-admissions market configuration that led to it.

\section{Early Admissions Equilibrium}

All the proofs of my results are deferred to Appendix A. College $j$ 's admissions problem is to choose an admission policy that maximizes its expected utility and exactly exhausts capacity. Given strategy profile $\Sigma_{\sigma_{j}}$, for admissions process $p$ and college $j$, I let $S_{j}^{p}\left(q ; \Sigma_{\sigma_{j}}\right)$ be the capacity expected to be occupied by applicants of quality $q$ if admitted and $X_{j}^{p}\left(q ; \Sigma_{\sigma_{j}}\right)$ the expected financial aid offer to be made to an enrolled student of quality $q$. Note that, conditional on strategy profile, $S_{j}^{p}$ and $X_{j}^{p}$ do not depend on college $j$ 's admission policy. ${ }^{10}$ For ease of exposition, I defer the formal

${ }^{10}$ This is an implication of the timing and rules of the game. As students can apply at most once to a given college, and as they do not observe the outcome of the early process before the regular one, conditional on a strategy profile, college $j$ 
construction of $S_{j}^{p}, X_{j}^{p}$, and the colleges' admission problem to Appendix A.1.

\subsection{Optimal Admissions Policy}

To determine the optimal admission policy, it is useful to look at the contribution to expected utility of the capacity expected to be filled by students of quality $q$ upon admission (CEU), which is the benefit-to-cost ratio of admitting the students of quality $q$. Conditional on strategy profile $\Sigma_{\sigma_{j}}$, if college $j$ admits its applicants with quality $q$ during process $p$, it gets expected utility of $S_{j}^{p}\left(q ; \Sigma_{\sigma_{j}}\right)\left[u(q)-\alpha_{j} X_{j}^{p}\left(q ; \Sigma_{\sigma_{j}}\right)\right] \geq 0 ;{ }^{11}$ meanwhile, admitting these students comes at an expected expense of $S_{j}^{p}\left(q ; \Sigma_{\sigma_{j}}\right)$ units of capacity. Therefore, the CEU is simply

$$
C_{j}^{p}\left(q ; \Sigma_{\sigma_{j}}\right) \equiv u(q)-\alpha_{j} X_{j}^{p}\left(q ; \Sigma_{\sigma_{j}}\right) \geq 0
$$

when $S_{j}^{p}\left(q ; \Sigma_{\sigma_{j}}\right)>0$, and zero otherwise.

I define a Generalized Admission Threshold Strategy (GATS) to be an admission policy in which a college admits only students with a CEU above a given threshold; at an intuitive level, a GATS is an admission strategy that prioritizes students per their benefit-to-cost ratio. Lemma 1 formalizes that when there are enough students to enroll, it is necessary and sufficient for the efficiency in the admission process to use a GATS that exhausts capacity:

Lemma 1. Consider a given strategy profile $\Sigma_{\sigma_{j}}$ in which college $-j$ enrolls at most $1-\kappa$ students. An admission policy is optimal for college $j$ if and only if it is a GATS that enrolls exactly $\kappa$ students, namely, there exists a threshold $C_{j}>0$ such that

(i) the college admits every student with a CEU above the threshold $C_{j}$ (i.e., $\sigma_{j}^{p}(q)=1$ when $\left.C_{j}^{p}\left(q ; \Sigma_{\sigma_{j}}\right)>C_{j}\right) ;$ and

(ii) the college rejects every student with a CEU below the threshold $C_{j}$ (i.e., $\sigma_{j}^{p}(q)=0$ when $\left.0<C_{j}^{p}\left(q ; \Sigma_{\sigma_{j}}\right)<C_{j}\right)$.

By considering financial aid in the colleges' problem, Lemma 1 implies a key difference of my model with previous work on college admissions (Avery and Levin, 2010; Chade et al., 2014; Lee, 2009; Nei and Pakzad-Hurson, 2018): in the aforementioned literature, colleges admit only the students with a quality above a given threshold (a quality-threshold admission strategy). If a college is significantly hurt by financial aid, an admission threshold in CEU might not coincide with

cannot affect its set of regular applicants or potential enrollees trough changes it its early-admissions policy or vice-versa. More detail is found in Appendix A.1's Remark 1.

${ }^{11} \mathrm{~A}$ number $S_{j}^{p}\left(q ; \Sigma_{\sigma_{j}}\right)$ of such applicants enroll, and each of them produces a reward in expected-utility of $u(q)-$ $\alpha_{j} X_{j}^{p}\left(q ; \Sigma_{\sigma_{j}}\right)$. The non-negativity follows from the assumption that $0=\alpha_{A}<\alpha_{B}<\frac{u(0)}{\bar{n}}$. 
one in quality: ${ }^{12}$ the college might reject relatively high-quality students in favor of lower-quality ones, to save in financial aid. I call this phenomenon justified envy. Justified envy has been studied before (Avery and Levin, 2010; Che and Koh, 2016) but its role as a consequence of financial considerations is a novel insight of this paper.

Given that (to my knowledge) there is no empirical evidence of justified envy at top private selective schools, and to focus attention on the financial aid and early admissions part of the game, I separately treat the theoretical issues related to justified envy in Section 5.3. Formally, I assume that college B's capacity and sensitivity to financial considerations are such that it is optimal to use a quality-threshold admission policy; ${ }^{13}$ this assumption greatly simplifies the exposition but has no effect on the existence, uniqueness, or properties of the equilibrium, as I discuss at the end of Section 5.3.

\subsection{Equilibrium}

Theorem 1. A unique EA equilibrium outcome with the following properties exists:

(i) College A uses REA and college B uses ED.

(ii) College A's financial aid policy covers full demonstrated need, whereas college B's covers weakly less (that is, $1=\rho_{A} \geq \rho_{B}$ ).

(iii) Both colleges use a quality-threshold admission policy with thresholds $\left(A_{E}, A_{R}\right)$ and $\left(B_{E}, B_{R}\right)$, such that $B_{E}<A_{E} \leq A_{R}<1$ and $B_{E}<B_{R}<1$; in other words, both colleges favor early applicants, and college $\mathrm{A}$ is more selective than college $\mathrm{B}$.

(iv) Low-income students apply early to college A's REA program.

(v) There is a taste level $Y>0$ such that high-income students apply early to college B if $y<Y$ and apply early to college A otherwise; in other words, high-income students do not always apply early to their top choice.

The general proof of Theorem 1 is involved so I present its main insights through the following particular case:

Particular-Case Setup. Students' quality, taste, and financial need are independent from each other, with $q \sim U[0,1], y \sim U\left[-\frac{1}{3}, \frac{2}{3}\right]$, and $\frac{1}{4}$ of the students being low-income. Low- and high-income students have financial need of $\bar{n}=1$ and $\underline{n}=0$, respectively, so colleges evaluate a low-income

\footnotetext{
12This claim is shown in Lemma 4 in Appendix A.

${ }^{13}$ Lemma 5 in Appendix A formalizes that a "large enough" $\kappa$ or a "small enough" $\alpha_{B}$ are sufficient for the optimality a capacity-exhausting quality-threshold admission policy.
} 
student's financial need to be $\hat{n}_{j} \sim U[0,2]$. Students discount enrolling regular at rate $\delta=0.75$, and high-income ones get utility from enrolling at each college of $v_{h}(A, y)=\frac{1}{2 / 3-y}$ and $v_{h}(B, y)=\frac{1}{1 / 3+y}$, respectively. Low-income students get expected utility from comparing financial aid offers of $\bar{v}\left(\rho_{B}\right)>2 \bar{v}_{A}\left(\rho_{B}\right)$ for any $\rho_{B}$. The quality component of colleges' utility function is $u(q)=q$. Colleges have capacity $\kappa=\frac{1}{3}$ and sensitivity to financial aid of $\alpha_{A}=0$ and $\alpha_{B}=0.1$, respectively.

I focus on the case where college A uses early (E) and regular (R) quality admission thresholds $\left(A_{E}, A_{R}\right)$ and college $\mathrm{B},\left(B_{E}, B_{R}\right){ }^{14}$ A simplifying observation is that college $\mathrm{A}$ has a strictly dominant strategy in covering full demonstrated need $\left(\rho_{A}=1\right)$ : increasing financial aid does not directly affect its utility through the expenditure but strictly increases its chances of enrolling low-income, high-quality students. ${ }^{15}$ Therefore, to show equilibrium behavior, it is enough to focus on $1=\rho_{A} \geq \rho_{B}$. For concreteness, I set $\rho_{B}=\frac{1}{2}$. This is without loss of generality because the following arguments apply whenever $\rho_{A} \geq \rho_{B}$.

Consider the case where college A offers REA and college B, ED. When college B offers ED, low-income students avoid it because of its binding commitment: a low-income student significantly values comparing financial aid $\left(\bar{v}\left(\rho_{B}\right)>2 \bar{v}_{A}\left(\rho_{B}\right)\right)$, so if she was admitted early at college $\mathrm{B}$, she would not be able to apply to college A at all. This implies that college B must benefit early applicants in equilibrium: to optimize, college B is indifferent between the marginal early and regular admitted student, i.e.,

$$
B_{E}-0.1 \cdot X_{B}^{E}\left(B_{E} ; \Sigma_{\sigma_{B}}\right)=B_{R}-0.1 \cdot X_{B}^{R}\left(B_{R} ; \Sigma_{\sigma_{B}}\right)
$$

however, as no low-income student applies early, college B knows that the average early applicant requires less financial aid than the average regular applicant, i.e., $X_{B}^{E}\left(B_{E} ; \Sigma_{\sigma_{B}}\right)<X_{B}^{R}\left(B_{R} ; \Sigma_{\sigma_{B}}\right)$, which implies that $B_{E}<B_{R}$.

As college A is more popular among high-income students and also has a better financial aid policy than college $\mathrm{B}$, it behaves more selectively, i.e., $B_{E}<A_{E}$. This, along with the fact that college B favors early applicants, attracts some high-income students with a weak preference for college A. These students are near indifferent between both colleges in terms of taste, so they prefer to increase their chances of securing a spot at college B, instead of taking a "long-shot" approach at college A. Formally, there is a unique taste level $Y>0$ indifferent between applying early to either

${ }^{14}$ The optimality of this admission policy is formally proved in Lemma 2 in Appendix A.

${ }^{15}$ This claim is formally proved in Lemma 3 in Appendix A. 
college, with satisfies

$$
\overbrace{\left(1-B_{E}\right) \frac{1}{1 / 3+Y}}^{\text {Applies early to B }}=\overbrace{\underbrace{\left(1-A_{E}\right) \frac{1}{2 / 3-Y}}_{\text {Admitted REA to A }}+\underbrace{0.75 \cdot\left(A_{E}-\min \left\{A_{E}, B_{R}\right\}\right) \frac{1}{1 / 3+Y}}_{\text {Rejected REA at A, admitted regular at B }}}^{\text {Applies early to A }},
$$

such that every high-income student with $y<Y$ prefers to apply early to college $\mathrm{B}$, and to college A if $y>Y$.

Finally, it must be that college A favors early applicants $\left(A_{E} \leq A_{R}\right)$ and low-income students apply early to its REA program. If college A did not favor early applicants, it would not receive early applications: low-income students would maximize their chance of getting two offers by applying regular, the high-income students with a strong preference for college A would maximize their chances of being admitted by applying regular, and the rest of the high-income students would apply early to college B out of impatience.

It can be seen that when college B uses ED and college A uses REA, there are unique $\left(Y, A_{E}, B_{E}, B_{R}\right)$ that solve Equations 1 and 2, exhaust both colleges' capacity, and lead to a unique sub-game equilibrium outcome — the early admissions outcome (EAO)—with the following properties: low-income students apply early to college A, high-income students with $y>Y \approx 0.18$ apply early to college A, and to college B otherwise, and colleges use the capacity-exhausting thresholds $B_{E} \approx 0.32<B_{R} \approx 0.34, B_{E} \leq A_{E} \approx 0.4 \leq A_{R}$. Panel b of Figure 1 depicts the admissions outcome for this case: the students in the blue (solid) region enroll at college $\mathrm{B}$, whereas the ones in the red (striped) region enroll at college A.

To see why in equilibrium college $\mathrm{B}$ and A must use ED and REA respectively, it is useful to derive the admissions outcome when colleges offer no early admissions. As more than half of the students prefer college A over B (it is more prestigious and $\rho_{A} \geq \rho_{B}$ ), it must be more selective than college B, i.e., $A_{R}>B_{R}$. From direct computation, it follows that there are unique capacityexhausting thresholds $B_{R}=\frac{1}{3}$ and $A_{R} \approx 0.52$, which lead to a unique regular-admissions-only outcome (RAO), depicted in Panel a of Figure 1: among students with $q>A_{R}$, the high-income that prefer college A enroll there, as well as more than half of the low income students. The rest of the students with $q>B_{R}$ enroll at college B.

By using ED and favoring early applicants, college B gains a competitive edge over college A, compared to the RAO-the competitive force. First, the use of an ED program turns the early admissions process into a wealth-screening device for college B-low-income students avoid it — which allows it to favor early applicants. Second, by favoring early applicants, college B enrolls high-income students that otherwise would enroll at college $\mathrm{A}$ - the students in area $c$ in Figure 
Figure 1: Example's Enrollment Patterns.

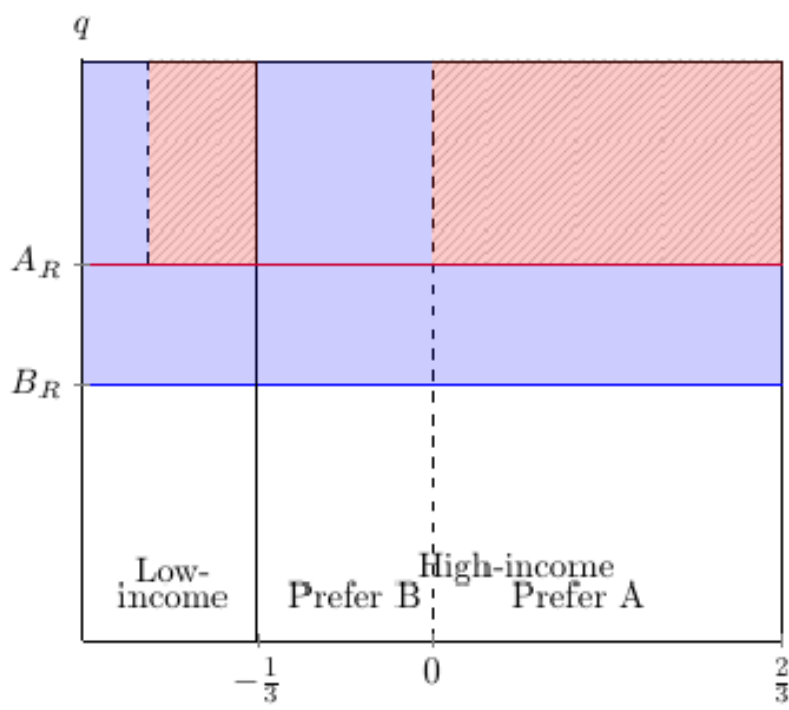

(a) Regular Admissions Only

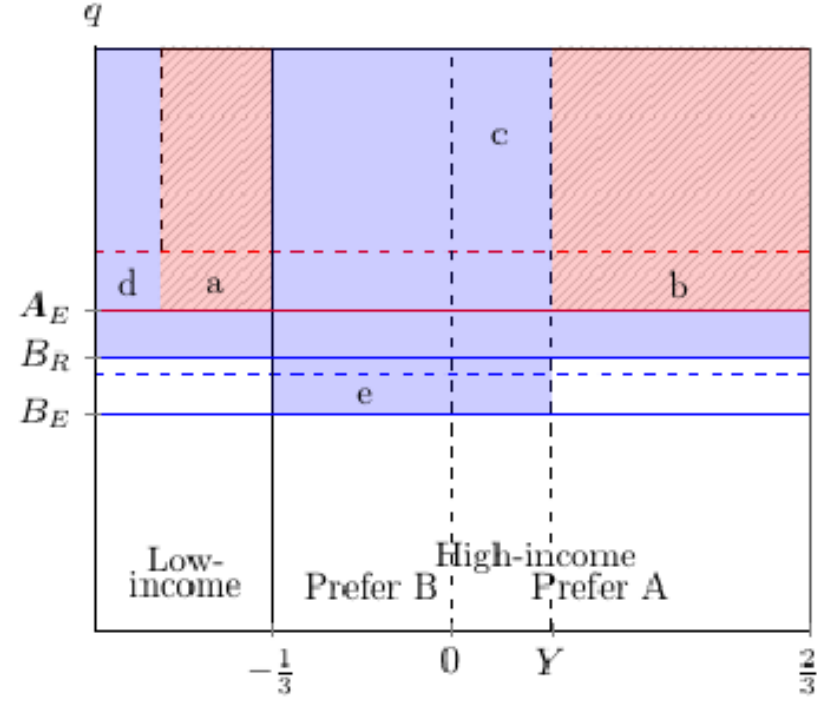

(b) Early Admissions

Note: The vertical axis measures the students' quality, whereas the horizontal one sorts the students by their income and taste. The admission thresholds are not drawn to scale. The blue (solid) area represents the enrolled students at college B, whereas the red (striped) area represents the ones enrolled at college A. The dashed horizontal lines in the early-admissions outcome represent the regular-admissions-only thresholds. $Y$ is the high-income student indifferent between applying early to college A and applying early to college B when early admissions are offered.

1. As a result, college A must behave less selective in the EAO, compared to the RAO: to exhaust capacity, it needs to lower its admission threshold and enroll the students in areas $a$ and $b$, which enroll at college B in the RAO. This exchange of students is unambiguously favorable for college B and unfavorable for college A: the former exchanges low- and high-income, lower-quality students (areas $a$ and $b$ ) for high-income, high-quality students (area $c$ ).

The competitive edge that college B gains over college A by using ED is what leads to the unique equilibrium early-admissions market configuration from Theorem 1. In a nutshell, college B can always induce the EAO by offering ED, whereas, by offering any other program, it induces an outcome similar to the RAO. Therefore, as college B prefers the former over the latter, it never has incentives to deviate from offering ED, and the unique early-admissions market configuration follows. The uniqueness of the EA equilibrium outcome follows from the fact that college B's expected utility is strictly concave in $\rho_{B}$, so a unique maximizer exists. ${ }^{16}$

16This claim is formally proved in Proposition 6 in Appendix A. 
Formally, in equilibrium, college A always offers REA. It never offers ED because its preferences are driven by student quality, so the low-income, high-quality students that avoid ED programs are valuable for it; meanwhile, it prefers to offer REA to no early admissions because the latter gives the high-income students with a preference for it an additional incentive to apply early to college B instead, out of impatience (area c of Figure 1 grows). Therefore, given that college A will offer REA, college B can induce the EAO by offering ED. If college B offered REA or no early admissions instead, both early programs would be non-binding and the equilibrium outcome would be similar to the RAO: every student can complete two applications and enroll at her preferred college if admitted to both. ${ }^{17}$

\subsection{Evidence}

In this section, I contrast the predictions of Theorem 1 (statements (i) to (iv) next) with data. The facts presented next were constructed with 2018 data from the Integrated Postsecondary Education Data System (IPEDS), the Common Data Set (CDS), the College Board (CB), US News and World Report (USN), and colleges' web pages (CWP). I also considered evidence from Avery et al. (2009), Avery and Levin (2010) and Avery et al. (2012). Details about my sources of information and variables can be found in Online Appendix D, whereas summary statistics can be found in Appendix B.

(i) College A uses REA and college B uses ED. In my model, college A is the most prestigious and wealthy college. In the data, one can distinguish among top private colleges by their choice of early-admissions format: Harvard, Stanford, Princeton, and Yale offer REA; University of Chicago offers both REA and ED; MIT, CalTech, and Georgetown offer an unrestricted version of early action; ${ }^{18}$ and the rest of the colleges offer ED (CB, CDS, USN). ${ }^{19}$ According to the USN general ranking, ${ }^{20}$ REA colleges rank better than ED colleges, with an average rank of 3.3 compared to 18.2. Likewise, according to a students' revealed-preference ranking estimated by Avery et al. (2012),

17These claims are proved in Propositions 2 to 5 in Appendix A.

${ }^{18}$ This form allows students to apply early to multiple colleges, as long as the other colleges allow it.

${ }^{19}$ The ED colleges are Boston University, Brandeis University, Brown, Carnegie Mellon, Columbia, Cornell, Dartmouth, Duke, Emory, Johns Hopkins, New York University, Northwestern, Rice, Tufts, University of Pennsylvania, Vanderbilt, and Washington University in St. Louis.

${ }^{20}$ The USN general ranking is based on a composite index that weights characteristics concerning academic quality, faculty resources, graduate performance, experts opinion, and financial resources, among others. The lower the index, the more attractive the college. 
the REA colleges have an average rank of 3.75, compared to ED colleges' average rank of 28.6. ${ }^{21}$ Furthermore, according to the USN financial-resources ranking, ${ }^{22}$ REA colleges rank, on average, 5.75, whereas ED colleges rank 21.5. REA colleges have about \$2 million of endowment per full-time equivalent student, compared with ED colleges' \$0.37 million (IPEDS).

(ii) College A's financial aid policy is better than college B's. In the data, REA colleges are more generous with their financial aid offers than ED colleges: all of the former cover full demonstrated need, whereas only 65 percent of latter do. Among need-blind colleges, despite a comparable average cost of attendance at both types of schools, low- and high-income students pay less at REA colleges: on average, low-income students pay $\$ 2,160$ a year at an REA college and $\$ 7,335$ at an ED one, whereas high-income ones pay $\$ 37,184$ at an REA college and \$43,334 at an ED one (USN). Furthermore, REA colleges offer about twenty-five times more scholarships and grants than repayable aid, compared to the twelve times that ED colleges do (USN).

(iii) Both colleges favor early applicants and college A is more selective than college B. In the data, both REA and ED colleges are very selective. However, the former are far more selective: they accept about 6 percent of their applicants, whereas ED colleges accept about 16 percent (IPEDS). Despite the high selectivity of these colleges, all else equal, applicants improve their admission chances by applying early, with ED applicants benefiting more than REA applicants: by applying early, applicants improve their chances of admission by about 31 to 37 percentage points at ED colleges and by about 17 to 20 points at REA colleges (Avery and Levin, 2010).

(iv) Low-income students avoid college B's ED, which (v) attracts high-income students with a preference for college $A$. The fact that low-income students are more likely to avoid ED's binding commitment than high-income ones is well documented by Avery et al. (2009) and Antecol and Kiholm Smith (2012); the former, for instance, reports that "Generally, ED applicants are a bit less needy than the overall pool $(50-55 \%$ vs. $60 \%)$...," whereas the latter finds evidence of students from disadvantaged backgrounds and minority groups being more likely to apply regular to colleges

${ }^{21}$ Avery et al. (2012) constructed the revealed-preference ranking with survey data on students' preferences for colleges. The lower the rank, the more preferred the college.

${ }^{22}$ The USN financial-resources ranking is based on a composite index that weights the expenditure per student by such characteristics as instruction, research, and student services and support. The lower the index, the more attractive the college. 
that offer ED, seeking for competing financial aid offers. Finally, Avery et al. (2009) and Avery and Levin (2010) provide evidence that students behave strategically and apply early to a college that is not their first choice.

\section{Welfare}

A common belief is that early-admissions programs benefit colleges and high-income students, whereas they hurt low-income ones (Avery et al., 2009; Chapman and Dickert-Conlin, 2012; Fetter, 1997; The Chronicle of Higher Education, 2006) . According to this belief, a low-income student's welfare is reduced because comparing financial aid offers is crucial for her and applying early reduces her chances of getting multiple financial aid offers. In contrast, a high-income student without financial concerns benefits from an increased likelihood of acceptance at her preferred college. Finally, colleges benefit from having more financial control over their early applicants, compared to the regular ones. I assess these claims in light of my model. I compare the EAO from Theorem 1 with the outcome of the counterfactual scenario in which colleges can only offer regular admissions, i.e., the RAO. Let $\rho_{B}^{*}$ be college B's optimal financial aid policy. Theorem 2 summarizes my findings:

Theorem 2. There exists $\bar{V}$ such that if $\bar{v}\left(\rho_{B}^{*}\right)>\bar{V}$ and $B_{R}<A_{E}$, when colleges offer early admissions, compared to the regular-admissions-only case,

(i) college B's welfare is strictly higher, whereas college A's is strictly lower;

(ii) the ex ante welfare of high-income students is strictly higher; and

(iii) the ex ante welfare of low-income students is strictly higher.

Theorem 2 establishes that if comparing financial aid is sufficiently important for low-income students $\left(\bar{v}\left(\rho_{B}^{*}\right)>\bar{V}\right)$ and college $\mathrm{B}$ is willing to enroll regular some of the top early applicants rejected by college $\mathrm{A}\left(B_{R}<A_{E}\right),{ }^{23}$ then college $\mathrm{B}$ and all students prefer early admissions, whereas college A prefers no early admissions. It is important to remark some aspects of the result. First, it is slightly more general than what Theorem 2 establishes: colleges' and high-income students' welfare is always true, independently of the conditional statement, as the proof shows. Second, the requirement that college $\mathrm{B}$ is willing to enroll regular some of the early applicants rejected at college

23"If $B_{R}<A_{E}$ " is a slight abuse of notation meaning "if $\alpha_{B}$ is 'small enough' or $\kappa$ is 'big enough' (or both) in a way that, in equilibrium, $B_{R}<A_{E}$." The existence of such $\kappa$ and $\alpha_{B}$ follows from Lemmas 4 and 5 in Appendix A. 
A (the wealthier and more prestigious school) is reasonable, given that the top rejected students at elite colleges are still very competitive among applicants at the rest of the schools. ${ }^{24}$

The main insights behind colleges' welfare result were examined in the previous section: the use of ED by college B induces an exchange of students that is favorable for it, but that hurts college A. Using the same example from previous section, I provide the main insights behind the welfare result for students. The welfare of high-income students is driven by a sorting effect more than by an increased chance of admission, contrary to popular belief. Thanks to early admissions, the students in areas $b$ and $e$ in Panel b of Figure 1 are better sorted (they enroll at their favorite college). These students, however, are among the ones with the strongest preferences for their favorite college, making their welfare improvement very significant. On average, and ex ante, this sorting effect is strong enough to drive the welfare of high-income students, who, as a result, strictly benefit from early admissions. This establishes part (ii) of Theorem 2.

When comparing financial aid offers is very important for low-income students, the low-income students in areas $d$ and $a$ in Panel b of Figure 1 drive their ex ante welfare. Because of the competitive force, which makes college A less selective, when early admissions are present, these students are admitted to both colleges and enroll at the one making the better of two offers, whereas when only regular admissions are present, they enroll at college B with only one offer. This establishes part (iii) of Theorem 2.

\section{Extensions}

\subsection{Students' Preferences}

Let $\tau \in\{l, h\}$ be an index representing a student's income and $x_{j}$ her financial aid offer at college $j$. Suppose a given student's utility utility from enrolling at college $j$ is $v_{\tau}\left(j, x_{j}, y\right)>0$, where $v_{\tau}\left(A, x_{A}, y\right)$ and $v_{\tau}\left(B, x_{B}, y\right)$ are strictly positive, continuous in $y$ and strictly increasing in $x_{A}$ and $x_{b}$ respectively. Furthermore, $v_{\tau}\left(A, x_{A}, y\right)$ and $v_{\tau}\left(B, x_{B}, y\right)$ are strictly increasing and decreasing, respectively, in $y$. The student with $y=0$ is indifferent between the two colleges, conditional on both offering the same amount of financial aid, so $v_{\tau}(A, x, 0)=v_{\tau}(B, x, 0)$ for any $x$. I assume that an extreme taste for a given college translates into a very strong preference — that is, $\lim _{y \rightarrow \bar{y}} v_{\tau}(A, x, y)=\lim _{y \rightarrow \underline{y}} v_{\tau}(B, x, y)=\infty$, for any $x$.

${ }^{24}$ Table 2 in Avery and Levin (2010) shows that, on average, the best colleges' early applicants have a stronger profile (for instance, SAT and high school quality) than the rest of the colleges' regular applicants. 
Let $\bar{v}^{\tau}\left(\rho_{B}\right)$ be the expected utility of the student with taste $y=0$ when she has an offer from both schools and $\underline{v}_{j}^{\tau}\left(\rho_{B}\right)$ be her expected utility when she only has an offer from college $j .{ }^{25}$ Then, $\frac{v_{A}^{l}\left(\rho_{B}\right)}{\delta G^{*}}<\bar{v}^{l}\left(\rho_{B}\right)$ guarantees that low-income student's preferences are mainly driven by getting two financial aid offers, whereas $\delta \bar{v}^{h}\left(\rho_{B}\right)<\underline{v}_{B}^{h}\left(\rho_{B}\right)$ guarantees that high-income student's preferences are mainly driven by taste. Under these restrictions, my results remain qualitatively unaffected:

Theorem 3. Under general students' preferences, if $\frac{\underline{v}_{A}^{l}\left(\rho_{B}\right)}{\delta G^{*}}<\bar{v}^{l}\left(\rho_{B}\right)$ and $\delta \bar{v}^{h}\left(\rho_{B}\right)<\underline{v}_{B}^{h}\left(\rho_{B}\right)$ for any $\rho_{B}$, a unique EA equilibrium outcome exists. This outcome has the same properties of Theorem 1 , except for $(i v)$, which is modified as follows:

(iv') There is a taste level $Y_{l}<0$ such that low-income students apply early to college B if $y<Y_{l}$ and apply early to college A otherwise.

Moreover, there exists $\bar{V}$ such that if $\bar{v}^{l}\left(\rho_{B}^{*}\right)>\bar{V}$ and $B_{R}<A_{E}$, the welfare properties in Theorem 2 are true.

The predictions of Theorem 1 are unaffected when students' general preferences are considered, except for the situation of low-income students. In Theorem 1, low-income students apply early to college A, whereas in Theorem 3, some apply early to college B-the ones with an extreme taste for it $\left(y<Y_{l}\right)$. The fact that $Y_{l}<0$ implies that some low-income students with a preference for college B are deterred from applying ED by the binding commitment of the program-the ones almost indifferent between colleges. This, in fact, is enough to make ED attractive for college B and to produce the incentives necessary to offer a lower admission threshold for early applicants: college B knows that early applicants are wealthier, in average, than regular ones. The rest of the arguments behind the result remain unaffected, including the ones for high-income students: as long as their main preference driver is taste, some of them with a taste for college A are not deterred from applying ED to college B to increase their admission chances. Finally, from a welfare perspective, my results remain unaffected and the arguments are symmetric to the ones behind Theorem 2 .

\subsection{College A's Preferences}

For ease of exposition, I assumed that college A's sensitivity to the expenditure in financial aid was zero $\left(\alpha_{A}=0\right)$. I show that my results extend to the case where college A's sensitivity to financial aid is positive but small. In this case, the intuition behind my previous arguments still hold true: college A significantly values student quality over financial aid, so it is interested in attracting

${ }^{25}$ Formally, $\bar{v}^{\tau}\left(\rho_{B}\right)=\mathbb{E}\left(\max \left\{v^{\tau}\left(A, x_{a}, 0\right), v_{\tau}\left(B, x_{b}, 0\right)\right\} \mid \rho_{A}=1, \rho_{B}, n, y=0\right)$ and $\underline{v}_{j}^{\tau}(0)=\mathbb{E}\left(v_{\tau}\left(j, x_{j}, 0\right) \mid \rho_{A}=\right.$ $\left.1, \rho_{B}, n, y=0\right)$. 
the low-income, high-quality students that avoid college B's ED program. Moreover, to maximize the proportion of such students, college A is interested in covering full demonstrated financial-need. Formally,

Theorem 4. There is $\bar{\alpha}>0$, such that if $\alpha_{A} \in[0, \bar{\alpha}]$, there exists a unique EA equilibrium outcome with the properties of Theorem 1. Moreover, there exists $\bar{V}$ such that if $\bar{v}^{l}\left(\rho_{B}^{*}\right)>\bar{V}$ and $B_{R}<A_{E}$, the welfare properties in Theorem 2 are true.

\subsection{Justified Envy}

In this section, I present additional insights about justified envy in the context of financial aid. To understand why justified envy arises, consider a strategy profile $\Sigma_{\sigma_{B}}$ in which both colleges offer only regular admissions and college A uses an admission threshold $A_{R}$ that enrolls at most $1-\kappa$ students. Lemma 1 implies that college B must set a capacity-exhausting threshold $C_{B}$ and admit only every student with a CEU above it.

Consider the applicants with $q=A_{R}$ and the ones with quality just below $A_{R}$. Both groups have almost the same quality, but the former is admitted to college A, whereas the latter is not. If college $\mathrm{B}$ admits the students with $q=A_{R}$, it knows that only the following will enroll: the high-income ones with $y<0$, and the low-income ones for whom college B's financial aid offer is better than college A's. If college B admits the ones with $q<A_{R}$, it knows that all of them will enroll, including the low-income ones that have no other admission offer.

As a result, an enrollee with $q=A_{R}$ is more likely to be low income than one with $q<A_{R}$. Furthermore, among low-income enrolled students, the ones with $q=A_{R}$ are, on average, more expensive than the ones with $q<A_{R}$ : the average enrolled student with $q=A_{R}$ requires more financial aid than the average one with $q$ just below $A_{R}$. If college $\mathrm{B}$ is hurt enough by financial aid commitments (see Lemmas 4 and 5), it could be the that this difference in the financial aid expenditure leads the college to reject higher-quality students in favor of lower-quality, cheaper ones. The scenario is depicted by the black CEU function in Figure 2.

To see that this disparity in expected expenditure is not sufficient for justified envy, in Figure 2 I show a CEU for $\alpha_{B}^{\prime}<\alpha_{B}$ (red dashed line), and an alternative CEU threshold $\bar{C}_{B}<C_{B}$ that responds to larger college capacity. Under both circumstances the GATS translates into a quality-threshold admission policy and justified envy is not present. Lemma 5 in Appendix A formalizes the existence of such an $\alpha_{B}$ and $\bar{C}_{B}$ for a general case.

Although justified envy is an interesting possibility, assuming that $\kappa$ and $\alpha_{B}$ are such that it 


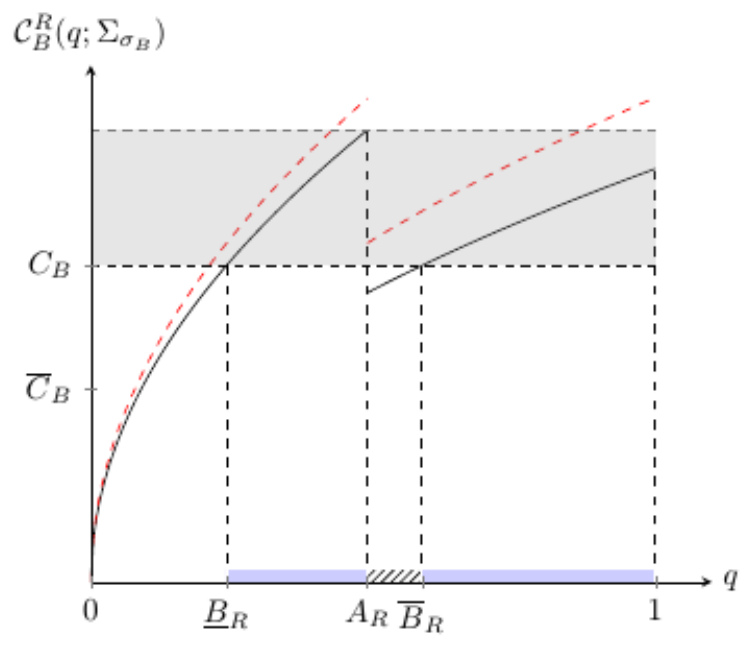

Figure 2: Justified envy.

Note: The vertical axis measures CEU units, and the horizontal one measures quality. The black function is a given CEU. The gray area represents the CEU levels admitted under the GATS, for a threshold $C_{B}$. The highlighted area over the horizontal axis represents the admitted quality levels under the GATS. The striped area on the horizontal axis represents the quality levels that suffer justified envy. The red-dashed function represents the black CEU after a ceteris paribus reduction in $\alpha_{B}$ that eliminates justified envy; likewise, $\bar{C}_{B}$ represents a $\mathrm{CEU}$ threshold reduction from $C_{B}$ that eliminates justified envy.

does not arise is of no consequence for my results. This is because the map whose fixed point is an equilibrium is based on the CEU thresholds directly, not on their translation into quality-based admission policies. In Murra-Anton (2019), I present an early version of my model and analyze it regardless of justified envy: the complexity of exposition is significantly greater, whereas the results are qualitatively the same.

\section{Concluding Remarks}

Through a novel college admissions model with financial aid, I showed how need-blind colleges compete using early admissions and how, through such programs, some of them can limit their financial commitments. One prediction of my analysis is that there exists a unique equilibrium outcome for the early admissions game, given the colleges' prestige, rank and financial situation. This seems consistent with the fact that the early admissions market configuration has remained stable for the past ten years, in which no significant changes in colleges' characteristics have happened.

The unique equilibrium outcome prediction might seem at odds, however, with all the market changes that took place when early programs first were implemented: colleges changed the format 
of early admissions they offered, or even eliminated their programs, without a significant change in their prestige, rank or endowment (Avery et al., 2009). Nonetheless, anecdotal evidence suggests that colleges did not know the full extent of early admissions consequences at first, and had to learn as they operated the programs.

For instance, the following quotes suggest that, at first, Harvard was not aware of the early admissions' competitive or diversity effects, and had to adjust accordingly as it discovered them: when in 2006 Harvard eliminated early admissions altogether (it had used ED), William Fitzsimmons, the dean of admissions and financial aid, explained: "Early applicants tended to be disproportionately white and affluent, and there was a growing perception that early admissions was becoming an exclusive club, to which somehow only a few were invited" (The Chronicle of Higher Education, 2006); then, in 2011, when Harvard restored early admissions in the form of REA, Michael Smith, the dean of the Faculty of Arts and Sciences, explained: "We looked carefully at trends in Harvard admissions these past years and saw that many highly talented students, including some of the best-prepared low-income and underrepresented minority students, were choosing programs with an early-action option, and therefore were missing out on the opportunity to consider Harvard" (Harvard University, 2011).

In my analysis, I assumed that colleges are need-blind. This was a reasonable assumption, given that I focused on top private selective colleges, which almost all claim to have need-blind policies. Under this assumption, my model satisfactorily explained important facts related to early admissions and financial aid. Nonetheless, many aspects of need-blind admissions remain in need of attention in future work. Bear in mind that need-blind admissions is a statutory requirement for colleges to participate in a mutually beneficial antitrust exemption granted by Congress. The objective of Congress was to expand college access for low-income students. More work is needed to fully understand the possible implications of need-blind admissions and to evaluate whether the incentives of the market are aligned with the government's. Additional aspects to study are, first, the factors driving the decision to offer need-blind admissions and, second, the competition between need-blind and other schools.

Finally, I showed that colleges' financial considerations could, in theory, lead to justified envy. To my knowledge, most of the empirical and theoretical work on college admissions focuses on quality-threshold admission policies, and none has studied whether justified envy is present in data; future empirical work on this topic is of great value for the understanding of the college admissions problem. 


\section{References}

Antecol, Heather and Janet Kiholm Smith, "The early decision option in college admission and its impact on student diversity," The Journal of Law and Economics, 2012, 55 (1), 217-249.

Ashby, Cornelia, "Higher Education: Schools' Use of the Antitrust Exemption Has Not Significantly Affected College Affordability or Likelihood of Student Enrollment to Date. Report to Congressional Committees," GAO-06-963, Government Accountability Office, 2006.

Avery, Christopher and Jonathan Levin, "Early admissions at selective colleges," American Economic Review, 2010, 100 (5), 2125-56.

_, Andrew Fairbanks, and Richard J Zeckhauser, The early admissions game, Harvard University Press, 2009.

_ , Mark E Glickman, Caroline M Hoxby, and Andrew Metrick, "A revealed preference ranking of us colleges and universities," The Quarterly Journal of Economics, 2012, 128 (1), 425-467.

Chade, Hector, Gregory Lewis, and Lones Smith, "Student portfolios and the college admissions problem," Review of Economic Studies, 2014, 81 (3), 971-1002.

Chapman, Gabrielle and Stacy Dickert-Conlin, "Applying early decision: Student and college incentives and outcomes," Economics of Education Review, 2012, 31 (5), 749-763.

Che, Yeon-Koo and Youngwoo Koh, "Decentralized college admissions," Journal of Political Economy, 2016, 124 (5), 1295-1338.

Epple, Dennis and Richard E Romano, "Competition between private and public schools, vouchers, and peer-group effects," American Economic Review, 1998, pp. 33-62.

_ , Richard Romano, and Holger Sieg, "Admission, tuition, and financial aid policies in the market for higher education," Econometrica, 2006, 74 (4), 885-928.

Fetter, Jean H, Questions and admissions: Reflections on 100,000 admissions decisions at Stanford, Stanford University Press, 1997.

Harvard University, "Early action returns," 2011.

Hill, Catharine B and Gordon C Winston, "Access: Net prices, affordability, and equity at a highly selective college," Economics of Education Review, 2006, 25 (1), 29-41.

Kim, Matthew, "Early decision and financial aid competition among need-blind colleges and universities," Journal of Public Economics, 2010, 94 (5-6), 410-420.

Lee, Sam-Ho, "Jumping the curse: Early contracting with private information in university admissions," International Economic Review, 2009, 50 (1), 1-38.

Murra-Anton, Zeky, "College Early Admissions: Determinants and Welfare," Available at SSRN 3451291, 2019.

Nei, Stephen and Bobak Pakzad-Hurson, "Strategic Disaggregation in Matching Markets," Available at SSRN 2523224, 2018.

The Chronicle of Higher Education, "Harvard Plans to End Policy of Admitting Students Early by Eric Hoover," 2006. 


\section{A Mathematical Constructions and Proofs}

\section{A.1 Colleges' Admission Problem}

I define $\mathcal{X}_{j}$ to be the space of college $j$ 's possible financial aid offers. In my model, the only information needed to characterize application, admissions, and enrollment decisions for a given student is her type $(q, y, n) \in \mathcal{T}$ and her financial aid offers $\left(x_{A}, x_{B}\right) \in \mathcal{X}_{A} \times \mathcal{X}_{B}$. The following interpretation is useful. Suppose that before applying, the student draws from $F$ a financial aid offer from each college but that neither the student nor the college observes the outcome of the draw until after the student is admitted: the student applies knowing only $(y, n)$, and the college admits her based only on $q$. Given that there is a unit measure of students behaving symmetrically, and their type and financial aid offers are independent of other students', one can think of an extended type $\tilde{t} \in \tilde{\mathcal{T}} \equiv \mathcal{T} \times \mathcal{X}_{A} \times \mathcal{X}_{B}$ as the representative student with those characteristics (that is, each extended type is one student). I let $\mathbb{I}(\theta)$ be the indicator function of event $\theta$. Based on this interpretation, I define several objects that are crucial to understanding how college $j$ makes admission decisions conditional on college $-j$ 's admission policy and students' application behavior:

Definition 3. Take a strategy profile $\Sigma_{\sigma_{j}}$. For a given college $j$ and a process $p$ :

- The set of applicants is 26

$$
\mathcal{A}_{j}^{p}\left(\Sigma_{\sigma_{j}}\right)=\left\{\tilde{t} \in \tilde{\mathcal{T}}: \tilde{t} \text { applies to } j \text { at process } p \text { under } \Sigma_{\sigma_{j}}\right\} .
$$

- The set of students with quality $q$ that would enroll if admitted is

$$
\mathcal{E}_{j}^{p}\left(q ; \Sigma_{\sigma_{j}}\right)=\left\{\tilde{t} \in \mathcal{A}_{j}^{p}\left(\Sigma_{\sigma_{j}}\right): \tilde{t} \text { has quality } q \text { and enrolls at } j \text { if she is admitted }\right\} .
$$

- The capacity expected to be occupied by applicants of quality $q$ that would enroll if admitted is $^{27}$

$$
S_{j}^{p}\left(q ; \Sigma_{\sigma_{j}}\right)=\mathbb{P}\left(\mathcal{E}_{j}^{p}\left(q ; \Sigma_{\sigma_{j}}\right)\right) .
$$

- The expected financial aid offer to be made to an enrolled student of quality $q$ is

$$
X_{j}^{p}\left(q ; \Sigma_{\sigma_{j}}\right)=\mathbb{E}\left(x_{j} \mathbb{I}\left(\mathcal{E}_{j}^{p}\left(q ; \Sigma_{\sigma_{j}}\right)\right)\right) .
$$

Remark 1. Under the rules of the game, students can apply at most once to a college. Accordingly, $\mathcal{A}_{j}^{p}$ and $\mathcal{E}_{j}^{p}$ do not depend on college $j$ 's admission policy.

${ }^{26}$ Note that as students do not observe $\left(q, x_{A}, x_{B}\right)$ at the moment of the application, if $\left(q^{\prime}, y^{\prime}, n^{\prime}, x_{A}^{\prime}, x_{B}^{\prime}\right) \in \mathcal{A}_{j}^{p}\left(\Sigma_{\sigma_{j}}\right)$, then $\left(q, y^{\prime}, n^{\prime}, x_{A}, x_{B}\right) \in \mathcal{A}_{j}^{p}\left(\Sigma_{\sigma_{j}}\right)$ for every $\left(q, x_{A}, x_{B}\right) \in[0,1] \times \mathcal{X}_{A} \times \mathcal{X}_{B}$.

${ }^{27}$ I use the term "applicants that would enroll if admitted" instead of "applicants that enroll" because colleges have the option to accept only a fraction of their applicants of quality $q$. 
To see why Remark 1 is true, consider the effect of early admissions on the regular process. By applying early to college $j$, a student waives her right to apply regular. Therefore, by accepting or rejecting an additional set of students during the early process-conditional on a strategy profile $\Sigma_{\sigma_{j}}$ - college $j$ cannot affect its set of regular applicants. Likewise, it cannot affect the set of applicants that would enroll if admitted regular because the game is simultaneous and students cannot modify their application strategy based on the early-admission policy. As a consequence, Definition 3 implies that $S_{j}^{R}$ and $X_{j}^{R}$ also are independent of college $j$ 's early-admission policy. A similar argument can be made for the effect of the regular admission policy on the early process.

To construct the college's admission problem, I highlight the following facts: A student that does not apply cannot be admitted. A student that is not admitted cannot enroll. A student that does not enroll cannot generate utility for the college. Fix a process $p$, a college $j$, and a strategy profile $\Sigma_{\sigma_{j}}$; observe that when $\mathcal{E}_{j}^{p}\left(q ; \Sigma_{\sigma_{j}}\right)=\emptyset$ or $\mathcal{A}_{j}^{p}\left(\Sigma_{\sigma_{j}}\right)=\emptyset$, Definition 3 implies that $S_{j}^{p}\left(q ; \Sigma_{\sigma_{j}}\right)=X_{j}^{p}\left(q ; \Sigma_{\sigma_{j}}\right)=0$ for every $q$.

I denote $\tilde{t}$ 's Bayes-consistent distribution by $H$, with density $h .^{28}$ The probability of being admitted, conditional on having applied and being of quality $q$, is $\mathbb{P}\left(\right.$ Admitted $\left.\mid q, \mathcal{A}_{j}^{p}\left(\Sigma_{\sigma_{j}}\right)\right)=$ $\sigma_{j}^{p}(q)$. In the event that $\mathcal{A}_{j}^{p}\left(\Sigma_{\sigma_{j}}\right)=\emptyset, \sigma_{j}^{p}(q)$ is defined to be zero: if there are no applicants, there are no students to admit. By Definition 3 and the facts noted above, $S_{j}^{p}\left(q ; \Sigma_{\sigma_{j}}\right)$ mathematically represents the probability of enrolling conditional on having quality $q$, having applied, and having being admitted. Likewise, $X_{j}^{p}\left(q ; \Sigma_{\sigma_{j}}\right)$ is the expected financial aid required by the student conditional on having quality $q$, having applied, having been admitted, and having enrolled. Therefore, the expected utility for the college of enrolling a student of quality $q$ is

$$
u(q)-\alpha_{j} X_{j}^{p}\left(q ; \Sigma_{\sigma_{j}}\right)
$$

The law of total expectation implies that the expected utility that the school gets from using the admission policy $\sigma_{j}(q)$ for its applicants of quality in a small neighborhood of $q$ is

$$
\left[u(q)-\alpha_{j} X_{j}^{p}\left(q ; \Sigma_{\sigma_{j}}\right)\right] \sigma_{j}^{p}(q) S_{j}^{p}\left(q ; \Sigma_{\sigma_{j}}\right) h\left(q \mid \mathcal{A}_{j}^{p}\left(\Sigma_{\sigma_{j}}\right)\right) d q,
$$

and, given the strategy profile $\Sigma_{\sigma_{j}}$, college $j$ 's expected utility of using admission policy $\sigma_{j}$ for its applicants is

$$
U_{j}\left(\sigma_{j}, \Sigma_{\sigma_{j}}\right) \equiv \sum_{p \in\{E, R\}} \int_{0}^{1}\left[u(q)-\alpha_{j} X_{j}^{p}\left(q ; \Sigma_{\sigma_{j}}\right)\right] \sigma_{j}^{p}(q) S_{j}^{p}\left(q ; \Sigma_{\sigma_{j}}\right) h\left(q \mid \mathcal{A}_{j}^{p}\left(\Sigma_{\sigma_{j}}\right)\right) d q .
$$

Following similar reasoning, given the strategy profile $\Sigma_{\sigma_{j}}$, college $j$ 's expected capacity filled

${ }^{28}$ In principle, $H$ could be the distribution under arbitrary beliefs. However, as, in my model, beliefs can always be computed through Bayes' rule, I proceed with the consistent distribution. 
by using admission policy $\sigma_{j}$ on its applicants is

$$
K_{j}\left(\sigma_{j}, \Sigma_{\sigma_{j}}\right) \equiv \sum_{p \in\{E, R\}} \int_{0}^{1} \sigma_{j}^{p}(q) S_{j}^{p}\left(q ; \Sigma_{\sigma_{j}}\right) h\left(q \mid \mathcal{A}_{j}^{p}\left(\Sigma_{\sigma_{j}}\right)\right) d q .
$$

With the construction above, I define College $j$ 's admissions problem as follows:

Definition 4. Given a strategy profile $\Sigma_{\sigma_{j}}$, college $j$ 's admissions problem is

$$
\begin{gathered}
\max _{\sigma_{j}} U_{j}\left(\sigma_{j}, \Sigma_{\sigma_{j}}\right), \\
\text { subject to } K_{j}\left(\sigma_{j}, \Sigma_{\sigma_{j}}\right)=\kappa .
\end{gathered}
$$

A different specification that leads to the same college admissions problem supposes that college $j$ 's ex post utility from enrolling a student of quality $q$ is $u(q)$, that the college has a budget for financial aid of $b_{j}>0$, and that each dollar of over- or underexpenditure on financial aid has a utility penalty or reward of $\alpha_{j} \geq 0$. The college's expected utility of using admission policy $\sigma_{j}$ - conditional on $\Sigma_{\sigma_{j}}$-is

$$
\begin{gathered}
\bar{U}_{j}\left(\sigma_{j}, \Sigma_{\sigma_{j}}\right)=\underbrace{\sum_{p \in\{E, R\}} \int_{0}^{1} u(q) \sigma_{j}^{p}(q) S_{j}^{p}\left(q ; \Sigma_{\sigma_{j}}\right) h\left(q \mid \mathcal{A}_{j}^{p}\left(\Sigma_{\sigma_{j}}\right)\right) d q}_{\text {Expected utility from students' quality }}+ \\
\alpha_{j}\left(b_{j}-\sum_{p \in\{E, R\}} \int_{0}^{1} X_{j}^{p}\left(q ; \Sigma_{\sigma_{j}}\right) \sigma_{j}^{p}(q) S_{j}^{p}\left(q ; \Sigma_{\sigma_{j}}\right) h\left(q \mid \mathcal{A}_{j}^{p}\left(\Sigma_{\sigma_{j}}\right)\right) d q\right)
\end{gathered}
$$

Expected reward or penalty from financial aid over- or under-expenditure

It is easy to see that $\bar{U}_{j}\left(\sigma_{j}, \Sigma_{\sigma_{j}}\right)=U_{j}\left(\sigma_{j}, \Sigma_{\sigma_{j}}\right)+b_{j} \alpha_{j}$, and given that $\alpha_{j} b_{j}$ is a constant, the problem in Definition 3 is equivalent to the one that considers $\bar{U}_{j}\left(\sigma_{j}, \Sigma_{\sigma_{j}}\right)$ instead of $U_{j}\left(\sigma_{j}, \Sigma_{\sigma_{j}}\right)$.

\section{A.2 Optimal Admission Policies}

Proof of Lemma 1. Fix a given strategy profile $\Sigma_{\sigma_{j}}$ under which college $-j$ enrolls at most $1-\kappa$ students.

-If $\sigma_{j}$ is optimal, then it is a GATS that exactly exhausts capacity. We show the result by a contra-positive argument. Consider a given policy $\sigma_{j}$. If it doesn't exhaust capacity, it cannot be optimal in the college admissions problem, and the result follows. Suppose, then, that $\sigma_{j}$ exhausts capacity but it is not a GATS. We construct a strictly profitable deviation to show that $\sigma_{j}$ cannot be optimal.

Note that a GATS that exhausts capacity always exists for college $j:$ as $\kappa<\frac{1}{2}$ and as college $-j$ enrolls at most $1-\kappa$ students, there are more than $\kappa$ students that are rejected by $-j$ that would 
enroll at $j$ upon admission. Then, college $j$ can rank them by $C_{j}^{p}\left(q, \Sigma_{\sigma_{j}}\right)$ and set $C>0$ such that by admitting all of those with $C_{j}^{p}\left(q, \Sigma_{\sigma_{j}}\right)>C$ and rejecting all those with $0<C_{j}^{p}\left(q, \Sigma_{\sigma_{j}}\right)<C$, it enrolls exactly $\kappa$ students. We call this GATS $\bar{\sigma}_{j}$.

We make two observations. First, as $\sigma_{j}$ and $\bar{\sigma}_{j}$ exhaust capacity, the students they differ on must account for the same number of seats. Second, as the definition of a GATS only restricts admission for students with $C_{j}^{p}\left(q, \Sigma_{\sigma_{j}}\right)>0, \sigma_{j}$ and $\bar{\sigma}_{j}$ must differ on students for which $C_{j}^{p}\left(q, \Sigma_{\sigma_{j}}\right)>0$, or else $\sigma_{j}$ is a GATS. Then, $\sigma_{j}$ must reject some students with $C_{j}^{p}\left(q, \Sigma_{\sigma_{j}}\right)>C$ or must admit some students with $0<C_{j}^{p}\left(q, \Sigma_{\sigma_{j}}\right)<C$ (possibly both). Other relevant possible differences among policies are the students with $C_{j}^{p}\left(q, \Sigma_{\sigma_{j}}\right)=C$, as the GATS definition does not specify a a particular admission policy for them. Formally, there are sets $S_{r}, S_{a}$, subsets of $\mathcal{A}_{j}^{E}\left(\Sigma_{\sigma_{j}}\right) \cup \mathcal{A}_{j}^{R}\left(\Sigma_{\sigma_{j}}\right)$, such that

- for every student in $S_{a}, 0<C_{j}^{p}\left(q ; \Sigma_{\sigma_{j}}\right) \leq C$ and $\sigma_{j}^{p}(q)>\bar{\sigma}_{j}^{p}(q)$;

- for every student in $S_{r}, C_{j}^{p}\left(q ; \Sigma_{\sigma_{j}}\right) \geq C$ and $\sigma_{j}^{p}(q)<\bar{\sigma}_{j}^{p}(q)$;

- there exists a strictly positive measure subset of $S_{a}$ with $C_{j}^{p}\left(q ; \Sigma_{\sigma_{j}}\right)>C$ and $\sigma_{j}^{p}(q)<1$ or a strictly positive measure subset of $S_{r}$ with $C_{j}^{p}\left(q ; \Sigma_{\sigma_{j}}\right)<C$ and $\sigma_{j}^{p}(q)>0$ (possibly both), and

$$
\begin{gathered}
\sum_{p \in\{E, R\}} \int_{S_{a}}\left(\sigma_{j}^{p}(q)-\bar{\sigma}_{j}^{p}(q)\right) S_{j}^{p}\left(q ; \Sigma_{\sigma_{j}}\right) h\left(q \mid \mathcal{A}_{j}^{p}\left(\Sigma_{\sigma_{j}}\right)\right) d q= \\
\sum_{p \in\{E, R\}} \int_{S_{r}}\left(\bar{\sigma}_{j}^{p}(q)-\sigma_{j}^{p}(q)\right) S_{j}^{p}\left(q ; \Sigma_{\sigma_{j}}\right) h\left(q \mid \mathcal{A}_{j}^{p}\left(\Sigma_{\sigma_{j}}\right)\right) d q .
\end{gathered}
$$

Equation 5 says that the students that $\sigma_{j}$ admits but $\bar{\sigma}_{j}$ rejects (left-hand side) must account for the same number of seats of the students that $\sigma_{j}$ rejects but $\overline{\sigma_{j}}$ accepts (right-hand side). By the construction of $S_{a}$ and $S_{r}$, however, equation 5 implies that

$$
\begin{gathered}
\sum_{p \in\{E, R\}} \int_{S_{a}}\left(\sigma_{j}^{p}(q)-\bar{\sigma}_{j}^{p}(q)\right) C_{j}^{p}\left(q ; \Sigma_{\sigma_{j}}\right) S_{j}^{p}\left(q ; \Sigma_{\sigma_{j}}\right) h\left(q \mid \mathcal{A}_{j}^{p}\left(\Sigma_{\sigma_{j}}\right)\right) d q< \\
\sum_{p \in\{E, R\}} \int_{S_{r}}\left(\bar{\sigma}_{j}^{p}(q)-\sigma_{j}^{p}(q)\right) C_{j}^{p}\left(q ; \Sigma_{\sigma_{j}}\right) S_{j}^{p}\left(q ; \Sigma_{\sigma_{j}}\right) h\left(q \mid \mathcal{A}_{j}^{p}\left(\Sigma_{\sigma_{j}}\right)\right) d q .
\end{gathered}
$$

Finally, as $C_{j}^{p}\left(q ; \Sigma_{\sigma_{j}}\right)=u(q)-\alpha_{j} X_{j}^{p}\left(q ; \Sigma_{\sigma_{j}}\right)$, the last equation implies that

$$
\begin{gathered}
\sum_{p \in\{E, R\}} \int_{S_{A}}\left(\sigma_{j}^{p}(q)-\bar{\sigma}_{j}^{p}(q)\right)\left[u(q)-\alpha_{j} X_{j}^{p}\left(q ; \Sigma_{\sigma_{j}}\right)\right] S_{j}^{p}\left(q ; \Sigma_{\sigma_{j}}\right) h\left(q \mid \mathcal{A}_{j}^{p}\left(\Sigma_{\sigma_{j}}\right)\right) d q< \\
\sum_{p \in\{E, R\}} \int_{S_{R}}\left(\bar{\sigma}_{j}^{p}(q)-\sigma_{j}^{p}(q)\right)\left[u(q)-\alpha_{j} X_{j}^{p}\left(q ; \Sigma_{\sigma_{j}}\right)\right] S_{j}^{p}\left(q ; \Sigma_{\sigma_{j}}\right) h\left(q \mid \mathcal{A}_{j}^{p}\left(\Sigma_{\sigma_{j}}\right)\right) d q .
\end{gathered}
$$

The left-hand side of equation 7 is the expected utility loss from the students that are rejected under $\bar{\sigma}_{j}$ but admitted under $\sigma_{j}$, whereas the right-hand side is the expected utility gain from the 
students that are admitted under $\bar{\sigma}_{j}$ but are rejected under $\sigma_{j}$. Equation 7 implies that $\bar{\sigma}_{j}$ is a strictly profitable deviation over $\sigma_{j}$, and therefore, the latter cannot be optimal.

-If $\sigma_{j}$ is a GATS that exactly exhausts capacity, it is optimal. Let $\bar{\sigma}_{j}$ be the capacity-exhausting GATS constructed in the previous part of this lemma, which always exists. By the arguments in the previous part, this $\bar{\sigma}_{j}$ is strictly preferred to any policy that is not a capacity-exhausting GATS. If $\bar{\sigma}_{j}$ is the only capacity-exhausting GATS, then, it must be an optimal admission policy.

If $\bar{\sigma}_{j}$ is not unique, we show that every other capacity-exhausting GATS leads to the same expected utility, making them all optimal. To see it, suppose that there exists $\sigma_{j} \neq \bar{\sigma}_{j}$, another capacity-exhausting GATS. We can focus on differences for students with $C_{j}^{p}\left(q, \Sigma_{\sigma_{j}}\right)>0$ only, as any differences for students with $C_{j}^{p}\left(q, \Sigma_{\sigma_{j}}\right)=0$ are payoff-immaterial (these students do not enroll upon admission). Note that $\sigma_{j}$ and $\bar{\sigma}_{j}$ must have the same threshold $C>0$ : if $C \neq \bar{C}$, say (without loss) because $C>\bar{C}$, either $\sigma_{j}$ under-enrolls or $\bar{\sigma}_{j}$ over-enrolls-both contradictions. Then, as $\sigma_{j}$ and $\bar{\sigma}_{j}$ have the same threshold and both exactly exhaust capacity, they must differ on the students for which $C_{j}^{p}\left(q, \Sigma_{\sigma_{j}}\right)=C$, in a way that the differences account for the same number of seats. This leads to an analogue of equation 5. Multiplying both sides of that equation by $C$ leads to an analogue of equation 6 that holds with equality. Finally, $C_{j}^{p}\left(q, \Sigma_{\sigma_{j}}\right)=C$ leads to an analogue of equation 7 which holds with equality. This implies that the differences between policies have no payoff implications, so that both $\sigma_{j}$ and $\bar{\sigma}_{j}$ have the same payoff.

\section{A.3 Proof of Theorem 1}

Proof of Theorem 1. In any PBE, colleges enroll $\kappa$ students and thus, Lemma 1 determines their optimal admission policy. The result is implied by Lemmas 2 and 3, along with Propositions 1 to 5; these results are shown in Appendix A.3.

To simplify the analysis, we can focus on the case where colleges have quality-threshold admission policies and $\rho_{A}$ : Lemma 2 shows that it is always optimal for college A to use a quality-threshold admission policy, and as a result, that it is also optimal for college B to use one; meanwhile, Lemma 3 establishes that college A has a strictly dominant strategy on using $\rho_{A}=1$, so that property (ii) of the result is true.

Then, the properties and existence of the EA equilibrium are shown by backwards induction. For each early market configuration and arbitrary $\rho_{B} \leq \rho_{A}=1$, Propositions 1 to 4 prove the existence and properties of each possible subgame EA equilibrium outcome. Proposition 2 shows that there is only one possible subgame EA equilibrium outcome-the early admissions outcome or $E A O$ - when $E_{B}=E D$, in which college A's best reply is to use $E_{A}=R E A$; Proposition 2 also 
shows that the EAO has properties (i), (iii), (iv) and (v) of the theorem's statement. Proposition 5 shows that college B strictly prefers the EAO to any other possible subgame EA equilibrium outcome, independently of $\rho_{B}$, so that, in equilibrium, $E_{B}=E D$ and $E_{A}=R E A$ is the only possibility. Finally, Proposition 6 shows that, for every possible subgame EA equilibrium outcome, there exists a unique $\rho_{B} \leq \rho_{A}=1$ that is optimal for college B.

\section{A.3.1 Equilibrium Outcome for Given Financial Aid and Early Admissions.}

Lemma 2. The following are true:

(i) When college B enrolls at most $1-\kappa$ students, it is always optimal for college A to exhaust its capacity through a quality-threshold admission policy with thresholds $A_{R}$ and $A_{E}$, the latter only existing when $E_{A} \neq \emptyset$.

(ii) if college A uses a quality-threshold admission policy that enrolls at most $1-\kappa$ of the students, it is also optimal for college $\mathrm{B}$ to use one as well with thresholds $B_{R}$ and $B_{E}$, the latter only existing when $E_{B} \neq \emptyset$.

(iii) Considering only quality-threshold admission policies is without consequence on the outcome or payoffs of the game.

Proof of Lemma 2. Part (i). Fix $\Sigma_{\sigma_{A}}$ such that college B enrolls at most $1-\kappa$ students. Lemma 1 implies that college A's optimal admission policy is a GATS that exhausts capacity. Note that $C_{A}^{p}\left(q ; \Sigma_{\sigma_{A}}\right)=u(q)$ for students with $q \in \mathcal{P}_{A}^{p}=\left\{q: S_{A}^{p}\left(q ; \Sigma_{\sigma_{A}}\right)>0\right\}$, provided $\alpha_{A}=0$. The GATS structure implies that, for the quality levels in $\mathcal{P}_{A}$, the college admits those with $u(q)>C_{A}$ and reject those with $u(q)<C_{A}$, for $C_{A}>0$ defined such that capacity is exactly exhausted. Given that $u$ is strictly increasing, among students with $q \in \mathcal{P}_{A}^{p}$, college A admits those with $q>u^{-1}\left(C_{A}\right) \equiv A_{p}$ and reject those with $q<A_{p}$.

Part (ii). Fix $\Sigma_{\sigma_{B}}$ such that college A uses a quality-admission threshold and enrolls at most $1-\kappa$ students. Consider college B's early applicants with $q \in \mathcal{P}_{B}^{E}=\left\{q: S_{B}^{E}\left(q ; \Sigma_{\sigma_{B}}\right)>0\right\}$. Note that $\left[0, A_{R}\right) \subset \mathcal{P}_{B}^{E}$ : every early applicant to college $\mathrm{B}$ that is rejected regular at college $\mathrm{A}$ would enroll at college B if admitted. After learning that $q$ is not admitted at college A regular, college B cannot learn anything else about the student's characteristics through the application and enrollment behavior other than what it can learn through $q$. Given that $q$ has an atomless distribution and is negatively affiliated with $n, X_{B}^{E}\left(q ; \Sigma_{\sigma_{B}}\right)$ is weakly decreasing and continuous for all $q<A_{R}$, and given that $u$ is strictly increasing, $C_{B}^{E}\left(q ; \Sigma_{\sigma_{B}}\right)$ is strictly increasing and continuous for all $q<A_{R}$.

Now consider the students with $q \geq A_{R}$. As students apply only observing a noisy signal of $q$, students cannot differentiate their enrollment behavior at college B by quality, only by admission 
status at college A. This implies that either each or none quality level $q \geq A_{R}$ is expected to have a strictly positive mass of enrollees, i.e., $\left[A_{R}, 1\right] \cap \mathcal{P}_{B}^{E} \in\left\{\emptyset,\left[A_{R}, 1\right]\right\}$. With this observation, for every $q \geq A_{R}$, either $C_{B}^{E}\left(q ; \Sigma_{\sigma_{B}}\right)>0$ is strictly increasing and continuous, by an argument analogous to the case of $q<q_{A}$, or $C_{B}^{E}\left(q ; \Sigma_{\sigma_{B}}\right)=0$.

By Lemma 1, among students with $q \in \mathcal{P}_{B}^{E}$, college B admits those with $u(q)-\alpha X_{B}^{E}\left(q ; \Sigma_{\sigma_{B}}\right)>$ $C_{B}$ and reject those with $u(q)-\alpha X_{B}^{E}\left(q ; \Sigma_{\sigma_{B}}\right)<C_{B}$, for $C_{B}>0$ defined such that capacity is exactly exhausted. Lemma 4 establishes, given that $C_{B}^{E}$ is increasing and almost-everywhere continuous in $q$, that college B's GATS translates into admitting only the early applicants with $q \in\left[\underline{B}_{E}, A_{R}\right) \cup\left[\bar{B}_{R}, 1\right]$; furthermore, Lemma 5 implies that if $\alpha$ is small enough—our assumption—and/or $\kappa$ is big enough, this GATS collapses into a quality-threshold admission policy with threshold $B_{E}$. A completely analogous argument can be made for college B's regular process.

Part (iii). As the students with $q \notin \mathcal{P}_{A}^{p}$ do not enroll upon admission, and the set of students with $q=A_{p}$ have measure zero, it is without payoff or outcome effect if college $\mathrm{A}$ admits or rejects them; therefore, it is without loss to consider college A's optimal admission policy to be the quality-threshold admission policy that admits every student with $q \geq A_{p}$ and rejects every student with $q<A_{p}$, until exactly exhausting capacity. A similar argument follows for college B.

Lemma 3. The unique optimal financial aid policy for College $\mathrm{A}$ is $\rho_{A}=1$.

Proof of Lemma 3. Fix the behavior of students and college B, such that it enrolls at most $1-\kappa$ students. For simplicity, suppose that colleges only offer regular admissions, as the argument is identical when early admissions are present. Lemma 2 implies that we can focus on the case where college A uses a quality-threshold admission policy. Suppose that $\rho_{A}<1$ is optimal and $A_{R}$ is college A's admission threshold. We construct a profitable deviation: if the college sets $1 \geq \rho_{A}^{\prime}>\rho_{A}$, and adjusts its admission threshold to $A_{R}^{\prime}>A_{R}$ to avoid over-enrollment-the proportion of low-income students enrolled is strictly increasing in $\rho_{A}$-it fills a strictly positive number of seats formerly occupied by students of quality $q \in\left[A_{R}, A_{R}^{\prime}\right)$ with students that have $q \geq A_{R}^{\prime}$. As college $\mathrm{A}$ is only concerned with students' quality, this feasible trade strictly increases its utility - a contradiction. Therefore, the only possible optimal financial aid policy for the college is $\rho_{A}=1$.

Proposition 1. Let $1=\rho_{A} \geq \rho_{B}$ and $E_{A}=E_{B}=\emptyset$. Then, there exists a unique subgame EA equilibrium outcome; in it, students apply to both colleges and colleges use quality-threshold admission policies with thresholds $A_{R}>B_{R}$. 
Proof of Proposition 1. Per Lemma 2, it is enough to consider EA equilibrium in which colleges admitting students per a quality-threshold admission policy with thresholds $A_{R}$ and $B_{R}$ set to exhaust capacity. As colleges cannot force regular applicants to enroll, students' preferences dictate enrollment. Given that students apply to both colleges, among the high-income ones, college A enrolls more than one half because $G_{y}(0 \mid q, n)>1 / 2$ for every $q$ and $n$. On the other hand, for any $\rho_{A} \geq \rho_{B}$, Lemma 6 implies that college A enrolls a proportion $P\left(\rho_{A}, \rho_{B}\right)=1-\frac{\rho_{B}}{2 \rho_{A}} \geq \frac{1}{2}$ of low-income students. Therefore, in any subgame EA equilibrium, college A enrolls strictly more than one-half of its admitted students. Thus, college B cannot exhaust capacity by setting $B_{R} \geq A_{R}$ and it must be the case that $B_{R}<A_{R}$. The uniqueness follows from the fact that $S_{j}^{R}\left(q ; \Sigma_{\sigma_{j}}\right)>0$ for every $q$, so that there exists unique thresholds $A_{R}$ and $B_{R}$ that exhaust colleges' capacity.

Proposition 2. Let $1=\rho_{A} \geq \rho_{B}$ and $E_{B}=E D$. Then, a unique subgame EA equilibrium outcome can arise. In it $E_{A}=R E A$ and:

(i) colleges admit students per a quality-threshold admission policy with thresholds $A_{E} \leq A_{R}<1$ and $B_{E}<B_{R}<1$, with $B_{E}<A_{E}$;

(ii) low-income students apply early to college A;

(iii) there is a unique $Y>0$ such that high-income students apply early to college A if $y>Y$ and apply early to college B otherwise.

Proof of Proposition 2. Lemma 2 and the EA equilibrium refinement imply that we can focus on the case where colleges use quality-threshold admission policies with thresholds $B_{E}<1, B_{R}<1$, $A_{E}<1$ and $A_{R}<1$, set to exhaust capacity at both colleges. Let $1=\rho_{A} \geq \rho_{B}, E_{B}=E D$, $\underline{v}_{j}(\rho)=\mathbb{E}\left(v_{l}\left(x_{j}\right) \mid n=\bar{n}, \rho_{j}\right)$ and $\bar{v}\left(\rho_{A}, \rho_{B}\right)=\mathbb{E}\left(\max \left\{v_{l}\left(x_{A}\right), v_{l}\left(x_{B}\right)\right\} \mid n=\bar{n}, \rho_{A}, \rho_{B}\right)$.

- Consider $E_{A}=R E A$. If a low-income student applies early to college $\mathrm{A}$ and takes the offer right away, she gets a payoff of $\left(1-G_{q}\left(A_{E} \mid n=\bar{n}, y\right)\right) \underline{v}_{A}(1)$. If the same student applies early to college A, regular to college B and takes the best of the two offers when admitted to both colleges, she gets a payoff of

$$
\begin{gathered}
\left(1-G_{q}\left(\max \left\{A_{E}, B_{R}\right\} \mid n=\bar{n}, y\right)\right) \delta \bar{v}\left(1, \rho_{B}\right)+\left(G_{q}\left(\max \left\{A_{E}, B_{R}\right\} \mid n=\bar{n}, y\right)-G_{q}\left(A_{E} \mid n=\bar{n}\right), y\right) \delta \underline{v}_{A}(1)+ \\
\left(G_{q}\left(\max \left\{A_{E}, B_{R}\right\} \mid n=\bar{n}, y\right)-G_{q}\left(B_{R} \mid n=\bar{n}\right), y\right) \delta \underline{v}_{B}(1)
\end{gathered}
$$

The former payoff is strictly bigger than the latter, so a low-income student admitted early to college A always waits to apply regular to college B before enrolling (Observation 1). This is true because $\bar{v}\left(\rho_{B}\right)>\frac{\bar{v}_{A}\left(\rho_{B}\right)}{\delta G^{*}}$ for every $\rho_{B}$, because in EA equilibrium, $\max \left\{A_{E}, B_{R}\right\}<\kappa$, so $\left(1-G^{*}\right)<\left(1-G_{q}\left(\max \left\{A_{E}, B_{R}\right\} \mid \bar{n}\right)\right)$, and because $\underline{v}_{j}\left(\rho_{j}\right) \geq 0$ for every $\rho_{j}$.

Observation 1 implies the following: 
1. In any subgame EA equilibrium, $A_{E} \leq A_{R}<1$. Suppose that there exists a subgame EA equilibrium with $A_{E}>A_{R}$. Note that no low-income student applies early to college A. Doing it, means that applying early to college B is not optimal. Then, as college A offers REA, if admitted, this student will not enroll until after applying regular to college $\mathrm{B}$, per Observation 1. If she waits and applies early nowhere, she maintains her chances of admission regular at college B unaffected, and strictly increases her chances at college A, which strictly improves her expected utility, so applying early to college A cannot be optimal in the first place.

As low-income students, then, apply regular to college A, $S_{A}^{R}\left(q ; \Sigma_{\sigma_{A}}\right)>0$ for every $q$ - the set of low-income students with a better offer from college B always are willing to enroll. Then, Lemma 1 implies that $C_{A}=C_{A}^{R}\left(A_{R} ; \Sigma_{\sigma_{A}}\right)=u\left(A_{R}\right)$, with $C_{A}$ the CEU threshold. On the other hand, the EA equilibrium refinement implies that there exists a strictly positive measure set of early applicants that would enroll if admitted, implying that $S_{A}^{E}\left(q ; \Sigma_{\sigma_{A}}\right)>0$ for every $q \geq A_{E}$ and thus, that $C_{A}^{E}\left(A_{E} ; \Sigma_{\sigma_{A}}\right)=u\left(A_{R}\right)=C_{A}$, by Lemma 2. However, $u$ is strictly increasing and $A_{E}>A_{R}$, which implies that $C_{A}=u\left(A_{R}\right)<u\left(A_{E}\right)=C_{A}$, a contradiction.

2. In any subgame EA equilibrium, $B_{E}<B_{R}<1$. Suppose that there exists a subgame EA equilibrium with $B_{E} \geq B_{R}$. Given that $A_{E} \leq A_{R}$ and $E_{A}=R E A$, no low-income student applies early to college B-they maximize their chance of being admitted to both colleges by applying early to college A. Under consistent beliefs, college B knows that early applicants are high-income, so that the marginally-enrolled early student is, on average, less expensive than the regular one, namely $X_{B}^{E}\left(B_{E} ; \Sigma_{\sigma_{B}}\right)<X_{B}^{R}\left(B_{R} ; \Sigma_{\sigma_{B}}\right)$; moreover, a reasoning similar to point 1's imply that $C_{B}^{R}\left(q ; \Sigma_{\sigma_{B}}\right)>0$ for every $q \geq B_{E}$ and $C_{B}^{E}\left(q ; \Sigma_{\sigma_{B}}\right)>0$ for every $q$. It follows then, by Lemma 1 , for the CEU threshold $C_{B}$,

$$
u\left(B_{R}\right)-\alpha_{B} X_{B}^{R}\left(q ; \Sigma_{\sigma_{B}}\right)=C_{b}=u\left(B_{E}\right)-\alpha_{B} X_{B}^{E}\left(B_{E} ; \Sigma_{\sigma_{B}}\right),
$$

which implies, as $u$ is strictly increasing, that $B_{E}<B_{R}-$ a contradiction.

3. In any subgame EA equilibrium, $A_{E}>B_{E}$. Suppose that there exists a subgame EA equilibrium with $A_{E} \leq B_{E}$. Given that $\rho_{A} \geq \rho_{B}$, every low-income student applies early to college A-its financial aid policy is more generous than college B's, its early program is nonbinding, it offers a better chance of admission to a college, and it offers a better chance of admission to both colleges. Likewise, every high-income student with $y>0$ applies early to college A because that maximizes the chances of enrolling at their favorite college. This implies, like in the proof of Proposition 1, that college A enrolls strictly more than one-half of the students with $q \geq A_{E}$, and makes it impossible for college B to exhaust capacity-a contradiction. 
-Students' optimal behavior. Fix $E_{B}=E D, E_{A}=R E A$. We focus on the case when $B_{E}<B_{R}<1$ and $B_{E}<A_{E} \leq A_{R}<1$, as no equilibrium can exist otherwise, per points 1-3 above. Note that high-income students always apply early: college A offers a better admission chance early than regular, with no binding commitment, so students prefer to apply early to college A than nowhere. For high-income students with $y<0$, applying early to college B is their optimal strategy-it maximizes their chances of being admitted at their preferred college. For a high-income student with $y>0$, a trade-off arises: if she applies early to college $\mathrm{B}$, she increases her chances of being admitted to any college, but she sacrifices expected utility, as college B's ED forces her to enroll at her least-favorite college.

Formally, if a high-income student with $y>0$ applies early to college A, she gets an expected utility of

$$
\left[1-G_{q}\left(A_{E} \mid y, \underline{n}\right)\right] v_{h}(A, y)+\left[G_{q}\left(A_{E} \mid y, \underline{n}\right)-G_{q}\left(\min \left\{A_{E}, B_{R}\right\} \mid y, \underline{n}\right)\right] \delta v_{h}(B, y),
$$

whereas if she applies early to college $\mathrm{B}$, she gets an expected utility of $\left[1-G\left(B_{E} \mid y, \underline{n}\right)\right] v_{h}(B, y)$. Therefore, she is indifferent between applying to college A or B when her taste is $y=Y$, for $Y$ such that

$$
\begin{gathered}
\frac{v_{h}(A, Y)}{v_{h}(B, Y)}= \\
1+\frac{\left.\left[G_{q}\left(A_{E} \mid Y, \underline{n}\right)-G_{q}\left(\min \left\{A_{E}, B_{R}\right\} \mid Y, \underline{n}\right)\right](1-\delta)+G_{q}\left(\min \left\{A_{E}, B_{R}\right\} \mid Y, \underline{n}\right)-G_{q}\left(B_{E} \mid Y, \underline{n}\right)\right)}{1-G_{q}\left(A_{E} \mid Y, \underline{n}\right)} .
\end{gathered}
$$

Note that $Y>0$ that solves equation 9 always exists and is unique. This owes to the following facts: $v_{h}(A, 0) / v_{h}(B, 0)=1$; the left-hand side of the equation is non-negative and strictly increasing in $Y$; the right-hand side is strictly bigger than one and it is strictly decreasing in $Y$, the latter due to the positive affiliation of $Y$ and $q$; and both sides of the equation are continuous, as $u$ is continuous and $G$ is atomless and full-support in $q$, for any $Y$ and $n$. From these properties, it follows that high-income students apply early to college A if $y>Y$ and to college B if $y<Y$.

Similar calculations for low-income students, based on Observation 1 and the fact that being admitted early to college B means having to enroll, we can see they apply early to college A only if

$$
\begin{array}{r}
\left(1-G_{q}\left(B_{E} \mid \bar{n}, y\right)\right) \underline{v}_{B}\left(\rho_{B}\right)< \\
\left(1-G_{q}\left(\max \left\{B_{R}, A_{E}\right\} \mid \bar{n}, y\right)\right) \delta \bar{v}\left(1, \rho_{B}\right)+ \\
\left(G_{q}\left(\max \left\{B_{R}, A_{E}\right\} \mid \bar{n}, y\right)-G_{q}\left(B_{R} \mid \bar{n}, y\right)\right) \delta \underline{v}_{B}\left(\rho_{B}\right)+ \\
\left(G_{q}\left(\max \left\{B_{R}, A_{E}\right\} \mid \bar{n}, y\right)-G_{q}\left(A_{E} \mid \bar{n}, y\right)\right) \delta \underline{v}_{A},
\end{array}
$$

which is true because $\bar{v}\left(\rho_{B}\right)>\frac{\bar{v}_{A}\left(\rho_{B}\right)}{\delta G^{*}}$ for every $\rho_{B}$, because in EA equilibrium, $\max \left\{A_{E}, B_{R}\right\}<\kappa$, so $\left(1-G^{*}\right)<\left(1-G_{q}\left(\max \left\{A_{E}, B_{R}\right\} \mid \bar{n}\right)\right)$, because $\underline{v}_{j}\left(\rho_{j}\right) \geq 0$ for every $\rho_{j}$, and because 
$\underline{v}_{B}\left(\rho_{B}\right) \leq \underline{v}_{A}\left(\rho_{B}\right)$, for every $\rho_{B}$.

-Colleges' optimal behavior. Suppose low-income students apply early to college A, along with high-income students with $y>Y>0$, whereas the rest of the students apply early to college B. As early applicants admitted to college B must enroll upon admission, $S_{B}^{E}\left(q ; \Sigma_{\sigma_{B}}\right)>0$ for all $q$; as no low-income student applies early, it is also true that $X_{B}^{E}\left(q ; \Sigma_{\sigma_{B}}\right)<X_{B}^{R}\left(q ; \Sigma_{\sigma_{B}}\right)$, for every $q$. Then, capacity-exhausting $B_{E}<B_{R}$ are optimal for college B when Equation 8 holds.

Given students' behavior and college B's optimal thresholds $B_{E}<B_{R}$, college A can only enroll students with $q>B_{E}$ early, and students with $q<B_{E}$ regular. If enrolling $P\left(1, \rho_{B}\right)$ of the low-income students and the high-income students with $y>Y$, all of quality $q>B_{E}$, is enough to fill capacity, $A_{E} \geq B_{E}$ such that college A exhausts capacity and any $u^{-1}\left(C_{A}\right)=A_{R} \geq B_{E}$ are optimal. Any $A_{R} \geq B_{E}$ is optimal because $S_{R}^{A}\left(q ; \Sigma_{\sigma_{A}}\right)=0$ for $q>B_{E}$, so Lemma 1 does not impose any additional restriction. Setting $A_{R}<B_{E}$ cannot be optimal, as it leads to a contradiction similar to the one in point 3 above.

If enrolling $P\left(1, \rho_{B}\right)$ of the low-income students and the high-income students with $y>Y$, all of quality $q>B_{E}$, is not enough to fill capacity, capacity-exhausting $A_{E}=A_{R}<B_{E}$ are the only optimal possibility for college A; this is true because $S_{A}^{p}\left(q ; \Sigma_{\sigma_{A}}\right)>0$ for every $q<B_{E}$ and every process, so that Lemma 1 implies that $u\left(A_{R}\right)=C_{A}=u\left(A_{E}\right)$.

-Equilibrium. We check that a self-map can be established, to look for an equilibrium through a fixed-point. From points 1-3 above, we know that no equilibrium can exist outside the case $B_{E}<B_{R}<1$ and $B_{E}<A_{E} \leq A_{R}<1$. We check that agents' best replies are consistent with each other for this case. When $B_{E}<B_{R}<1$ and $B_{E}<A_{E} \leq A_{R}<1$, students' optimal behavior is that low-income and high-income students with $y>Y>0$ apply to college A early, and to college B otherwise, for $Y$ determined per Equation 9.

Now, when students follow this optimal behavior, college B must set $B_{E}<B_{R}$ through Equation 8 , which is consistent with the case of interest. The consistency of college A's optimal behavior is not straightforward as there is one case that might lead to $A_{E}=A_{R}<B_{E}$, as analyzed above. However, this case will be ruled out by the fact that colleges exhaust capacity. When $A_{E}<B_{E}$, it can be checked that the trade-off for high-income students leading to Equation 9 reverts, producing a $Y^{\prime} \leq 0$ such that low-income students apply early to college B only if $y \leq Y^{\prime}$, whereas low-income students still apply early to college A (in equation $9, Y=1$ if $B_{E}=A_{E}=A_{R}$ ). Therefore, college A enrolls more than half of the students with $q \geq A_{E}\left(P\left(1, \rho_{B}\right) \geq \frac{1}{2}\right.$ of the low-income students (Lemma 6) and the high-income ones with $y>Y^{\prime}$ ). This, however, makes it impossible for college B to exhaust capacity with $B_{E} \geq A_{E}$ and must set $B_{E}<A_{E}$-both colleges have the same capacity. 
Finally, as $A_{R} \geq B_{E}$ is optimal for college $\mathrm{B}$, if is possible to set $A_{R} \geq A_{E}$ arbitrarily to achieve consistency.

So a self-map from $\left(A_{E}, B_{E}, B_{R}, Y\right)$ into itself can be established. Now, $A_{E}, B_{E}$ and $B_{R}$ are functions of the CEU $C_{A}$ and $C_{B}$, respectively, and they are determined such that colleges exhaust capacity from equation 4 :

$$
\kappa=\int_{A_{E}\left(C_{A}\right)}^{1} S_{A}^{E}\left(q ; \Sigma_{\sigma_{A}}\right) h\left(q \mid \mathcal{A}_{A}^{E}\left(\Sigma_{\sigma_{A}}\right)\right) d q
$$

and

$$
\kappa=\int_{B_{E}\left(C_{B}\right)}^{1} S_{B}^{E}\left(q ; \Sigma_{\sigma_{B}}\right) h\left(q \mid \mathcal{A}_{B}^{E}\left(\Sigma_{\sigma_{B}}\right)\right) d q+\int_{B_{R}\left(C_{B}\right)}^{1} S_{B}^{R}\left(q ; \Sigma_{\sigma_{B}}\right) h\left(q \mid \mathcal{A}_{B}^{R}\left(\Sigma_{\sigma_{B}}\right)\right) d q .
$$

A subgame EA equilibrium, then, must be a tuple $\left(C_{A}, C_{B}, Y\right)$ that simultaneously solves Equations 9, 11 and 12; it is convenient to think about this system of equations as the map $\Phi\left(C_{A}, C_{B}, Y\right)=\left(C_{A}\left(C_{B}, Y\right), C_{B}\left(C_{A}, Y\right), Y\left(C_{A}, C_{B}\right)\right)$, which already was shown to be a self-map. This map is also defined in a non-empty, compact and convex euclidean subset, as bound college capacities imply that $C_{j} \in[\underline{C}, \bar{C}]$, and as $Y \in[0, \bar{y}]$.

The continuity of $u$ implies that $A_{E}=u^{-1}\left(C_{A}\right)$ is continuous in $C_{A}$. Likewise, Equation 8 implies that $B_{E}$ and $B_{R}$ are continuous in $C_{B}$. Thus, Equation 9 implies that $Y$ is continuous in $C_{A}$ and $C_{B}$. Finally, $C_{j}$ is also continuous in $C_{-j}$ and $Y$. To see it, first note that $S_{j}^{p}\left(q ; \Sigma_{\sigma_{j}}\right)$ is continuous in $Y$ for admitted students: among admitted students, $S_{B}^{E}\left(q ; \Sigma_{\sigma_{B}}\right)=1$ due to ED's binding commitment. Under consistent beliefs, for $q \geq A_{E}$, college $\mathrm{B}$ understands that it only enrolls, in the regular process, low-income students for which $x_{B} \geq x_{A}$; letting $\mathbb{P}(n=\bar{n} \mid q)=P_{L}(q)$ and relying on Lemma $6, S_{B}^{R}\left(q ; \Sigma_{\sigma_{B}}\right)=P_{L}(q) \frac{\rho_{B}}{2 \rho_{A}}$, when $q \geq A_{R}$. If $q \in\left[\min \left\{B_{R}, A_{E}\right\}, A_{E}\right)$, college $B$ knows that it enrolls all the low-income students, as well as the high-income ones that applied to college A early but were rejected, so that $S_{B}^{R}\left(q ; \Sigma_{\sigma_{B}}\right)=P_{L}(q)+\left(1-P_{L}(q)\right)\left(1-G_{y}(Y \mid q, \underline{n})\right)$. Thus, as $G_{y}$ is atomless, $S_{B}^{p}\left(q ; \Sigma_{\sigma_{B}}\right)$ is continuous in $Y$. From a completely analogous argument, it follows that $S_{A}^{p}\left(q ; \Sigma_{\sigma_{A}}\right)$ is continuous in $Y$ as well. The distribution $H$ is continuous on $y$ and $q$ as well: it is a distribution that depends on $y$ and $q$ at most through $G$ - so it is continuous in $q$-and it is conditioned by an events of the form $\{Y<y\}$ and $\{Y>y\}$, due to high-income students' application behavior, so its absolute continuity implies its continuity on $y$, through Bayes Rule. As the integral operator is continuous on its limits of integration, equations 11 and 12 imply the continuity of $C_{j}$ on $C_{-j}$ and $Y$. We conclude that $\Phi$ is a continuous map.

$$
\text { As } S_{A}^{E}\left(q ; \Sigma_{\sigma_{A}}\right)>0 \text { for every } q \geq A_{E}, S_{B}^{p}\left(q ; \Sigma_{\sigma_{B}}\right)>0 \text { for every } q \geq B_{p} \text {, and } H\left(q \mid \mathcal{A}_{j}^{p}\left(\Sigma_{\sigma_{j}}\right)\right)
$$
is full-support, there exists a unique $C_{A}\left(C_{B}, Y\right)$ that makes equation 11 hold, as well as a unique $C_{B}\left(C_{A}, Y\right)$ that makes equation 12 hold. Likewise, as argued in the construction of equation 9, 
$Y\left(C_{A}, C_{B}\right)$ is unique. Therefore, $\Phi$ is single-valued, and thus it is non-empty, convex and closed. It follows from Kakutani's Fixed Point Theorem that a fixed point exists.

To see that the fixed point $\left(C_{A}, C_{B}, Y\right)$ is unique, suppose another fixed point $\left(C_{A}^{\prime}, C_{B}^{\prime}, Y^{\prime}\right)$ exists. If $Y^{\prime}>Y$, given the optimal application behavior, college A must set $C_{A}^{\prime}<C_{A}$ so that $A_{E}^{\prime}<A_{E}$, or else under-enrolls. As the left-hand side of equation 9 is strictly increasing in $Y$ and the numerator of the right-hand side is nondecreasing in $A_{E}$, it must be that $C_{B}^{\prime}<C_{B}$, so that $B_{E}^{\prime}<B_{E}$ and $B_{R}^{\prime}<B_{R}$. However, as at $\left(C_{A}, C_{B}, Y\right)$ college B enrolls $\kappa$ students, at $\left(C_{A}^{\prime}, C_{B}^{\prime}, Y^{\prime}\right)$ it must enroll strictly more than $\kappa$, a contradiction. An analogous argument shows that $Y^{\prime}<Y$ is not possible either. Thus, we must have $Y^{\prime}=Y$. A similar argument using equation 9 shows that if $C_{A}^{\prime}>C_{A}$, to keep its left-hand side constant, we must have $C_{B}^{\prime}>C_{B}$, which leads to a total market enrollment of less than $2 \kappa$ students, a contradiction; an analogous argument shows that $C_{A}^{\prime}<C_{A}$ is not possible either. Thus $C_{A}^{\prime}=C_{A}$. If $Y^{\prime}=Y$ and $C_{A}^{\prime}=C_{A}$, as $C_{B}\left(C_{A}, Y\right)$ is single-valued, we have $C_{B}^{\prime}=C_{B}$, so that both fixed points are the same, a contradiction. Thus, no other fixed point can exist.

A unique equilibrium outcome must exist when $\rho_{A}=1 \geq \rho_{B}$ and $\left(E_{A}, E_{B}\right)=(R E A, E D)$, as the fixed point of $\Phi$ is unique. This equilibrium outcome has properties (i), (ii) and (iii). Note that it is not a unique equilibrium as $A_{R}$ is free. To see that no EA equilibrium exists with $E_{A} \neq R E A$, we analyze the other two possibilities for college A:

- If $E_{B}=E D$ and $E_{A}=\emptyset$, through a proof analogous to the case of $E_{A}=R E A$, there exists a unique subgame EA equilibrium outcome. In it, the low-income students apply early nowhere, and the high-income student indifferent between applying early to college B or nowhere has a taste $Y^{\prime}>Y>0$. This is true because students discount enrolling regular, so college A not offering an early program presents additional incentive for the high-income students with a weak preference for college A to apply early to college B.

Letting $A_{E}$ be college A's early-admission policy when $E_{A}=R E A$, note that by using no early admissions instead of REA, college A loses the students with $y \in\left[Y, Y^{\prime}\right]$ and $q \geq A_{E}$, and must replace them with strictly lower-quality students. As $\alpha_{A}=0$, college A is strictly better-off using REA—quality fully determines college A's payoff.

- If $E_{B}=E D, E_{A}=E D$, through a proof analogous to the case of $E_{A}=R E A$, it can be seen that low-income students apply early nowhere. This implies that $S_{A}^{R}\left(q ; \Sigma_{\sigma_{A}}\right)>0$ for every $q$. As EA equilibrium refinement implies that a set of students apply early to college A and a strictly positive measure subset enrolls, $S_{A}^{E}\left(A_{E} ; \Sigma_{\sigma_{A}}\right)>0$, for $A_{E}$ college A's early threshold. Lemma 1 and $\alpha=0$ imply, then, that $A_{E}=A_{R}$. This implies that only high-income students with $y>Y>0$ apply early to college $\mathrm{A}$, and the rest to college $\mathrm{B}$, for some $Y$. This application pattern is the same as in the 
case $E_{A}=R E A$, so a similar argument shows that there is a unique subgame EA equilibrium with the same enrollment patterns than that case. However, as no low-income student applies early to college A, the tie-breaking rule favors $E_{A}=R E A$.

Proposition 3. Let $1=\rho_{A} \geq \rho_{B}$. There does not exist a subgame EA equilibrium in which $E_{B}=R E A$.

Proof of Proposition 3. As implied by Lemma 2, we can focus on the case where colleges use quality-threshold capacity-exhausting strategies with thresholds $B_{E}<1, B_{R}<1, A_{E}<1$ and $A_{R}<1$; meanwhile, Lemma 3 allows us to focus on $1=\rho_{A} \geq \rho_{B}$ and $E_{B}=R E A$. Suppose a subgame EA equilibrium exists.

On the one hand, $B_{E}>B_{R}$ leads to a contradiction:

1. If $B_{E}>B_{R}$, then no low-income student applies early to college $B$. If they do, it means that they do not find it optimal to apply early to college A. However, by applying early nowhere, they strictly increase their chances of admission at college B, without affecting their chances at college A. This translates into a strict increase in expected utility (as in Proposition 2, REA-admitted low-income students wait for the regular process to enroll) - a contradiction.

2. If no low-income student applies early to college $B, B_{E}<B_{R}$. If low-income students do not apply early to college $\mathrm{B}$, they apply regular. As a strictly positive measure set of early applicants enroll at college $\mathrm{B}, S_{B}^{p}\left(q ; \Sigma_{\sigma_{B}}\right)>0$, and thus $X_{B}^{E}\left(q ; \sigma_{\sigma_{B}}\right)<X_{B}^{R}\left(q ; \sigma_{\sigma_{B}}\right)$, so Equation 8 implies that $B_{E}<B_{R}$.

On the other hand, $B_{E} \leq B_{R}$ leads to a contradiction too:

3. If $B_{E} \leq B_{R}$, college $A$ offers REA with admission thresholds $A_{E}=A_{R}$. If college A offers ED or no early admissions, $B_{E} \leq B_{R}$ along with $\bar{v}\left(\rho_{B}\right)>\frac{\bar{v}_{A}\left(\rho_{B}\right)}{\delta G^{*}}$ makes it optimal for low-income students to apply early to college B. If low-income students apply early to college $\mathrm{B}, X_{B}^{E}\left(B_{E} ; \Sigma_{\sigma_{B}}\right)>X_{B}^{R}\left(B_{R} ; \Sigma_{\sigma_{B}}\right)$ and equation 8 implies that $B_{E}>B_{R}$, which is not possible. Finally, as a positive measure set of students apply early to college B but are rejected, they would enroll to college A regular if admitted; from Lemma 1, it follows that $u\left(A_{E}\right)=C_{A}=u\left(A_{R}\right)$, for $C_{A}$ the CEU threshold, which implies that $A_{E}=A_{R}$.

4. If college A offers REA with admission thresholds, $A_{E}>B_{R}$ and low-income students apply early to college $B$. An argument similar to the one in point 3 of Proposition 2's proof imply that college $\mathrm{A}$ is more selective than college $\mathrm{B}$ in equilibrium, namely, $A_{E}>B_{R}$. Thus, as $A_{E}=A_{R}$, low-income students maximize their chances of being admitted to both colleges by applying early to college B. 
5. If low-income students apply early to college $B$ and $A_{E}>B_{R}$, then $B_{E}>B_{R}$. If $A_{E}>B_{R}$, there is a group of students with $q \in\left[B_{R}, A_{E}\right]$ that applies early to college $\mathrm{A}$, is rejected and apply regular to college $\mathrm{B}$. These students are only high-income, so $X_{B}^{R}\left(B_{R} ; \Sigma_{\sigma_{B}}\right)<X_{B}^{R}\left(B_{E} ; \Sigma_{\sigma_{B}}\right)$, and thus, equation 8 implies that $B_{E}>B_{R}$.

Thus, no equilibrium can exist because if one did would be incompatible with both $B_{E} \leq B_{R}$ and $B_{E}>B_{R}$.

Proposition 4. Let $1=\rho_{A} \geq \rho_{B}$. When $E_{B}=\emptyset$ and $E_{A} \neq \emptyset$, there is a unique EA-equilibriumoutcome, the "reverse early-admissions outcome," in which $B_{R}<A_{E} \leq A_{R}<1$, $\frac{\rho_{B}}{2 \rho_{A}}$ of the low-income students and the high-income students with $y<Y$, for some $Y<0$, both with $q \geq A_{E}$, enroll at college $\mathrm{B}$, the rest of the students with $q \geq A_{E}$ enrolls at college $\mathrm{A}$, and every student with $q \in\left[B_{R}, A_{E}\right)$ enrolls at college $\mathrm{B}$.

Proof of Proposition 4. Let $1=\rho_{A} \geq \rho_{B}$ and $E_{B}=\emptyset$. As implied by Lemma 2, we can focus on the case where colleges use capacity-exhausting quality thresholds $B_{R}<1, A_{E}<1$ and $A_{R}<1$.

- If $E_{A}=R E A$, as in Propositions 2 and 3, in any subgame EA equilibrium, it must be that $A_{E}>B_{R}$, or college B does not fill capacity. It also must be true that $A_{E} \leq A_{R}$ as, if $A_{E}>A_{R}$, given that $\bar{v}\left(\rho_{B}\right)>\frac{\bar{v}_{A}\left(\rho_{B}\right)}{\delta G^{*}}$, low-income students apply early nowhere, $S_{A}^{R}\left(q ; \Sigma_{\sigma_{A}}\right)>0$ for every $q$ and it must be that $A_{E}=A_{R}-$ a contradiction (see the case of $E_{A}=E D$ in the proof of Proposition 2). The EA equilibrium refinement implies that $A_{R}<1$.

If $B_{R}<A_{E} \leq A_{R}<1$, every high-income student with $y>0$ applies early to college A. For high-income students with $y<0$, a trade-off arises: applying early to college A and saving in time discounting, at the expense of enrolling at their least-preferred college. As in the construction of equation 9, there is $Y<0$, such that if $y<Y$, the high-income student applies early to college A and enrolls if admitted, and if $y \in[Y, 0]$, the high-income student is indifferent between applying early to college A or B. Finally, low-income students apply early to college A if $A_{E}<A_{R}$ and are indifferent if $A_{E}=A_{R}$, but end up applying to both colleges, regardless (see Observation 1 of Proposition 2's proof). A fixed-point argument as in Proposition 2 implies that there exists a unique subgame EA equilibrium outcome with the following enrollment pattern: $\frac{\rho_{B}}{2 \rho_{A}}$ of the low-income students (see Lemma 3) and the high-income students with $y<Y$, for some $Y<0$, both with $q \geq A_{E}$, enroll at college $\mathrm{B}$, the rest of the students with $q \geq A_{E}$ enrolls at college $\mathrm{A}$, and every student with $q \in\left[B_{R}, A_{E}\right)$ enrolls at college B.

- If $E_{A}=E D$, like in the case of $E_{A}=R E A$ of this proof, $B_{R}<A_{E} \leq A_{R}<1$. The assumption that $\bar{v}\left(\rho_{B}\right)>\frac{\bar{v}_{A}\left(\rho_{B}\right)}{\delta G^{*}}$ implies that low-income students apply regular to college A-they avoid college A's ED. As in the case of $E_{A}=E D$ from the proof of Proposition 2, it follows that 
$A_{E}=A_{R}$. As students discount enrolling regular, there exists $Y<0$-as in the case of $E_{A}=R E A$ of this proof-such that high-income students with $Y<y$ apply early to college A, and wait for the regular process otherwise. A fixed-point argument as in Proposition 2 implies that there exists a unique subgame EA equilibrium outcome with the following enrollment pattern: $\frac{\rho_{B}}{2 \rho_{A}}$ of the low-income students (see Lemma 3) and the high-income students with $y<Y$, for some $Y<0$, both with $q \geq A_{E}$, enroll at college $\mathrm{B}$, the rest of the students with $q \geq A_{E}$ enrolls at college A, and every student with $q \in\left[B_{R}, A_{E}\right)$ enrolls at college B.

\section{A.3.2 Early Admissions Programs for Given Financial Aid Policy}

Proposition 5. Let $1=\rho_{A} \geq \rho_{B}$. Then college B strictly prefers the unique EA subgame equilibrium outcome that arises when $E_{B}=E D$, over any other possible subgame equilibrium outcome.

Proof of Proposition 5. Let $1=\rho_{A} \geq \rho_{B}$. Then, Propositions 1 to 4 hold. Among the possible subgame EA equilibrium outcomes of the game, we call the "Early Admissions Outcome" or EAO the one when $E_{B}=E D$ (c.f. Proposition 2), the "Regular-Admissions-Only Outcome" or RAO the one when $E_{A}=E_{B}=\emptyset$ (c.f. Proposition 1), and the "reverse EAO" or rEAO the one when $E_{B}=\emptyset$ and $E_{A} \neq \emptyset$ (c.f. part (ii) of Proposition 4). We denote by $J_{p}^{o}$ college $J$ 's admission threshold at process $p$ under outcome $o$. Let $Y^{E A O}$ be the EAO indifferent high-income student from Proposition 2, and $Y^{r E A O}$ be the rEAO indifferent one from Proposition 4.

- EAO vs. RAO. Note that, for fixed financial aid policies, the proportion of low-income students that each college enrolls, among those admitted to both colleges, is the same. Also note that in the EAO, college A enrolls only high-income students with $y>Y^{E A O}>0$, whereas in the RAO it enrolls the ones with $y>0$. As college A exhausts capacity in both, it must be that $A_{R}^{R A O}>A_{E}^{E A O}$.

We define $\Sigma_{\sigma_{B}}^{E A O}$ to be the situation in which everyone but college B, uses their subgame EAO optimal behavior, for $1=\rho_{A} \geq \rho_{B}\left(E_{A}=R E A, E_{B}=E D, A_{R}^{E A O}\right.$, low-income students apply early to college $\mathrm{A}$, and high-income ones apply early to college A only if $y>Y>0$ ). If college B uses $B_{E}=B_{R}=B_{R}^{R A O}$ as a response to $\Sigma_{\sigma_{B}}^{E A O}$, the following happen: (i) college B enrolls the high-income students with $q \geq A_{R}^{R A O}$ and $y \in\left[0, Y^{E A O}\right.$ )—which it does not in the RAO; (ii) college B does not enroll the low- and high-income students with $y>Y^{E A O}$, all of them with $q \in\left[A_{E}^{E A O}, A_{R}^{R A O}\right)$-which it does in the RAO; (iii) the rest of the students enroll the same in both scenarios. Note that the number of seats in (i) and (ii) must be the same: college A exhausts capacity in both EAO and RAO, and no other student changes enrollment status. This implies that college B exhausts capacity as well by using $B_{E}=B_{R}=B_{R}^{R A O}$, as a response to $\Sigma_{\sigma_{B}}^{E A O}$. 
College B is strictly better off, compared to the RAO, when it uses $B_{E}=B_{R}=B_{R}^{R A O}$ as a response to $\Sigma_{\sigma_{B}}^{E A O}$. To see it, note that the only difference for college B in enrollment between scenarios are the students in (i) and (ii). The ones in (i) are only high-income, whereas the ones in (ii) are both high- and low-income. Furthermore, each student in (i) has strictly bigger quality than every student in (ii). As both (i) and (ii) account for the same number of seats, getting the students in (i) strictly increases college B's expected utility.

- EAO vs. rEAO. College B strictly prefers the EAO to the rEAO and it is shown by an analogous argument using the high-income students with $q \geq A_{R}^{r E A O}$ and $y \in\left[Y^{r E A O}, Y^{E A O}\right)$ instead of the ones in (i), and the low- and high-income students with $y>Y^{E A O}$, all of them with $q \in\left[A_{E}^{E A O}, A_{R}^{r E A O}\right]$.

\section{A.3.3 Financial Aid Policy}

Proposition 6. At any subgame EA equilibrium outcome, there exists a unique financial aid policy $\rho_{B} \leq 1$ that maximizes college B's payoff.

Proof of Proposition 6. Lemma 3 implies that we can fix $\rho_{A}=1$. Fix the outcome from Proposition 1 first, in which colleges use admission thresholds $A_{R}>B_{R}$ and students apply to both colleges.

Note that when $\rho_{B}$ changes, the net effect on $B_{R}$ is zero: to see it, consider (without loss) an increase in $\rho_{B}$. Then, college B takes a subset of the low-income students with $q \geq A_{R}$ from college A. College A, as a result, has to make a reduction in $A_{R}$ to fill fill those seats: as $A_{R}>B_{R}$, this is possible by taking some of the students of low-income and high-income with $y>0$ that before the adjustment enrolled at college $\mathrm{B}$ because college $\mathrm{A}$ rejected them, i.e. those with $q \in\left[B_{R}, A_{R}\right)$. College A can always make up for a change in $\rho_{B}$ in a way that college B does not need to modify $B_{R}$ because $G_{y}(0 \mid q, \underline{n})>\frac{1}{2}$ for all $q$ and $\rho_{A}=1$ imply that even if $\rho_{B}=1, B_{R}<A_{E}$.

Then, given that $B_{R}$ does not depend on $\rho_{B}$, from Equation 3, we can write college B's payoff that account for optimal admissions as a function of $\rho_{B}$ :

$$
U\left(\rho_{B}, \Sigma_{\rho_{B}}\right)=\int_{B_{R}}^{1}\left[u(q)-\alpha_{B} X_{B}^{R}\left(q ; \Sigma_{\sigma_{B}}\right)\right] S_{B}^{R}\left(q ; \Sigma_{\sigma_{B}}\right) h\left(q \mid \mathcal{A}_{B}\left(\Sigma_{\sigma_{B}}\right)\right) d q .
$$

If $U\left(\rho_{B}, \Sigma_{\rho_{B}}\right)$ is continuous in $\rho_{B}$, as $\rho_{B} \in[0,1]$, the extreme value theorem implies that a maximizer exists. If, furthermore, $U\left(\rho_{B}, \Sigma_{\rho_{B}}\right)$ is strictly concave, the maximizer is unique. We show that indeed $U$ has these two properties. First, note that as students apply to both colleges, $h\left(q \mid \mathcal{A}_{B}^{R}\left(\Sigma_{\sigma_{B}}\right)\right)$ is independent of $\rho_{B}$ - the financial aid policy bears no information on the student's quality. We look at $U\left(\rho_{B}, \Sigma_{\rho_{B}}\right)$ 's partial integrals in $\left[A_{R}, 1\right]$ and $\left[B_{R}, A_{R}\right] .{ }^{29}$

\footnotetext{
${ }^{29} \mathrm{By}$ "partial integral" in $[a, b]$ we mean the integral on the right-hand side of equation 13 from $a$ to $b$.
} 
Consider students with $q \geq A_{R}$. The students that enroll at college B are only the low-income ones with $x_{B} \geq x_{A}$ and the high-income ones with $y<0$. As $\rho_{A} \geq \rho_{B}$, relying on Lemma 6, for $P_{L}(q)=\mathbb{P}(n=\underline{n} \mid q)$, we have that $S_{B}^{R}\left(q ; \Sigma_{\sigma_{B}}\right)=P_{L}(q) \frac{\rho_{B}}{2 \rho_{A}}+\left(1-P_{L}(q)\right) G_{y}(0 \mid q, \underline{n})$ and $S_{B}^{R}\left(q ; \Sigma_{\sigma_{B}}\right) X_{B}^{R}\left(q ; \Sigma_{\sigma_{B}}\right)=P_{L}(q) \frac{2\left(\rho_{B}\right)^{2}}{3 \rho_{A}} \bar{n}+\left(1-P_{L}(q)\right) G_{y}(0 \mid q, \underline{n}) \rho_{B} \underline{n}$. Notice that $S_{B}^{R}\left(q ; \Sigma_{\sigma_{B}}\right)$ is linear and continuous in $\rho_{B}$, whereas $S_{B}^{R}\left(q ; \Sigma_{\sigma_{B}}\right) X_{B}^{R}\left(q ; \Sigma_{\sigma_{B}}\right)$ is strictly convex and continuous in $\rho_{B}$. As a result, given $u(q)>0$ and $\alpha_{B}>0,\left(u(q)-\alpha_{B} X^{R}\left(q ; \Sigma_{\sigma_{B}}\right)\right) S_{B}^{R}\left(q ; \Sigma_{\sigma_{B}}\right)$ is strictly concave and continuous in $\rho_{B}$ for $q \geq A_{R}$. Given that integrand of equation 13, for every $q$, is finite with a finite first derivative, the partial integral in $\left[A_{R}, 1\right]$ is strictly concave and continuous in $\rho_{B}$ as well. ${ }^{30}$

Now consider the students with $B_{R} \leq q<A_{R}$. Among these students, all enroll at college B: therefore, $S_{B}\left(q ; \Sigma_{\sigma_{B}}\right)=1$ and $X_{B}\left(q ; \Sigma_{\sigma_{B}}\right)=\rho_{B}\left(P_{H} \bar{n}+\left(1-P_{L}(q)\right) \underline{n}\right)$, which is linear and continuous in $\rho_{B}$. As a result, $\left(u(q)-\alpha X\left(q ; \Sigma_{\sigma_{B}}\right)\right) S_{B}\left(q ; \Sigma_{\sigma_{B}}\right)$ is linear and continuous in $\rho_{B}$ for $B_{R} \leq q<A_{R}$. We conclude, as before, that the partial integral in $\left[B_{R}, A_{R}\right]$ is linear in $\rho_{B}$.

The fact that the partial integral is strictly concave in a strictly positive-measure subset of the integration region, and linear in the rest, implies that $U\left(\rho_{B}, \Sigma_{\sigma_{B}}\right)$ is strictly concave in $\rho_{B}$. Finally, as the integrand in equation 13 is continuous for every $q \geq B_{R}$ and the integral operation preserves continuity, $U\left(\rho_{B}, \Sigma_{\sigma_{B}}\right)$ is continuous in $\rho_{B}$ as well. The proof for the EAO and the rEAO is completely analogous.

\section{A.3.4 Auxiliary Results}

Lemma 4. If college A uses quality-threshold admission policy that enrolls at most $1-\kappa$ of the students, with thresholds $A_{E} \leq A_{R}$, it is optimal for college $\mathrm{B}$ to admit the early applicants with $q \in\left[\underline{B}_{E}, A_{R}\right) \cup\left[\bar{B}_{E}, 1\right]$ and the regular applicants with $q \in\left[\underline{B}_{R}, A_{E}\right) \cup\left[\hat{B}_{R}, A_{R}\right) \cup\left[\bar{B}_{R}, 1\right]$, for some $\underline{B}_{E} \leq A_{R} \leq \bar{B}_{E}$ and $\underline{B}_{R} \leq A_{E} \leq \hat{B}_{R} \leq A_{R} \leq \bar{B}_{R}$. Furthermore, considering only this type of strategy for college $B$ is without consequence on the outcome or payoffs of the game.

Proof of Lemma 4. Fix a strategy profile $\Sigma_{\sigma_{A}}$ in which college A uses a quality-threshold policy with thresholds $A_{E} \leq A_{R}$ and enrolls less than $1-\kappa$ students. Lemma 1 implies that it is optimal for college B to use a GATS. From Lemma $2, C_{B}^{E}\left(q ; \Sigma_{\sigma_{B}}\right)$ and $C_{B}^{R}\left(q ; \Sigma_{\sigma_{B}}\right)$ are strictly increasing and continuous in $q \neq A_{R}$ and $q \notin\left\{A_{R}, A_{E}\right\}$, respectively. Depending on $\kappa$ and $\alpha_{B}$, multiple cases could arise regarding the CEU threshold $C_{B}$.

Consider the early process. Let $A_{R}^{-}$be the left-limit of $q$ tending to $A_{R}$. Let $\underline{C}=C_{B}^{E}\left(A_{R}^{-} ; \Sigma_{\sigma_{B}}\right)$. If $\underline{C} \leq C_{B}^{E}\left(A_{R} ; \Sigma_{\sigma_{B}}\right)$, the CEU has at most a increasing discontinuity and $C_{B}^{E}\left(A_{R} ; \Sigma_{\sigma_{B}}\right)$ is strictly

${ }^{30}$ If $h(s)$ is strictly concave in $s$, finite and with a finite first derivative, $H(s)=\int_{a}^{b} h(s) d s$ is strictly concave as well: the Dominated Convergence Theorem implies that $H^{\prime \prime}(s)=\int_{a}^{b} h^{\prime \prime}(s) d s<0$, given that $h^{\prime}(s)<0$ for every $s$. 
increasing in for every $q$. In this case, college B-similar to college A's case in Lemma 2-uses a quality-threshold policy for early students with threshold $B_{E}$. In the notation of this Lemma, $\underline{B}_{E}=A_{R}$ and $\bar{B}_{E}=\left(C_{B}^{E}\right)^{-1}\left(C_{B} ; \Sigma_{\sigma_{B}}\right)$ if $\bar{B}_{E} \geq A_{R}$, whereas $\bar{B}_{E}=A_{R}$ and $\underline{B}_{E}=\left(C_{B}^{E}\right)^{-1}\left(C_{B} ; \Sigma_{\sigma_{B}}\right)$ if $\underline{B}_{E}<A_{R}$.

If $\underline{C}>C_{B}^{E}\left(A_{R} ; \Sigma_{\sigma_{B}}\right)$, the CEU has a decreasing discontinuity that can lead to justified envy. If there is a decreasing discontinuity and $C_{B}^{E}\left(A_{R} ; \Sigma_{\sigma_{B}}\right) \geq C_{B}$, no justified envy arises, as the monotonicity and continuity properties of the CEU imply that $\underline{B}_{E}=\left(C_{B}^{E}\right)^{-1}\left(C_{B} ; \Sigma_{\sigma_{B}}\right)<A_{R}$, and that every $q \geq \underline{B}_{E}$ has $C_{B}^{E}\left(A_{R} ; \Sigma_{\sigma_{B}}\right)>C_{B}$; then, we just set $\bar{B}_{E}=A_{R}$. Likewise if there is a decreasing discontinuity, $C_{B}^{E}\left(1 ; \Sigma_{\sigma_{B}}\right)>C_{B}$ and $\underline{C}<C_{B}$, so that $\bar{B}_{E}\left(C_{B}^{E}\right)^{-1}\left(C_{B} ; \Sigma_{\sigma_{B}}\right) \geq A_{R}$; then, we just set $\underline{B}_{E}=A_{R}$.

If there is a decreasing discontinuity, $C_{B}^{E}\left(A_{R} ; \Sigma_{\sigma_{B}}\right)<C_{B}$ and $\underline{C}>C_{B}$, justified envy arises: if it is the case that $C_{B}^{E}\left(1 ; \Sigma_{\sigma_{B}}\right)<C_{B}$ college $\mathrm{B}$ avoids every student with $q \geq A_{R}$ by setting $\underline{B}_{R}=$ $\left(C_{B}^{E}\right)^{-1}\left(C_{B} ; \Sigma_{\sigma_{B}}\right)<A_{R}$ and $\bar{B}_{R}=1$, whereas if it is true that $C_{B}^{E}\left(1 ; \Sigma_{\sigma_{B}}\right)>C_{B}$, the students with high-enough quality above $A_{R}$ are admitted, as well as some lower-quality students that are rejected at college A: formally, there exists $\underline{B}_{R}<A_{R}<\bar{B}_{R}$ such that $C_{B}^{E}\left(\underline{B}_{R} ; \Sigma_{\sigma_{B}}\right)=C_{B}^{E}\left(\bar{B}_{R} ; \Sigma_{\sigma_{B}}\right)=C_{B}$.

The case of regular admissions is proved in a completely analogous way, with the difference that now college B is affected by college A's early and regular admission policies. For that reason, the optimal GATS translates into a triple interval quality-based admission policy.

Lemma 5. Suppose colleges have capacity $\kappa_{A}$ and $\kappa_{B}$ with $\kappa_{A}+\kappa_{B}<1$. Fix a strategy profile $\Sigma_{\sigma_{B}}$ in which college $\mathrm{A}$ uses quality-thresholds $A_{E} \leq A_{R}$ and enrolls less than $1-\kappa_{B}$ students. Then, there exists $\kappa^{*}<1-\kappa_{A}$ and $\alpha^{*} \in(0,1)$ such that if $\kappa_{B} \geq \kappa^{*}$ or $0<\alpha_{B}<\alpha^{*}$, college B's optimal admission policy is always a quality-threshold policy. ${ }^{31}$

Proof of Lemma 5. Fix $\Sigma_{\sigma_{B}}$ in which college A uses quality thresholds $A_{E} \leq A_{R}$ and enrolls less than $1-\kappa_{B}$ students. If at $\kappa_{B}$ and $\alpha_{B}$ college B's admission policy is a quality-thresholds one (see the proof of Lemma 4 for details), the result is trivially true. We focus on the justified-envy case then, as established in the proof of Lemma 4.

Lemma 4 implies that it is optimal for college B to admit the early applicants with $q \in$ $\left[\underline{B}_{E}, A_{R}\right) \cup\left[\bar{B}_{E}, 1\right]$ and the regular applicants with $q \in\left[\underline{B}_{R}, A_{E}\right) \cup\left[\hat{B}_{R}, A_{R}\right) \cup\left[\bar{B}_{R}, 1\right]$, for some thresholds $\underline{B}_{E} \leq A_{R} \leq \bar{B}_{E}$ and $\underline{B}_{R} \leq A_{E} \leq \hat{B}_{R} \leq A_{R} \leq \bar{B}_{R}$. From the proof of Lemma 4 one can see that such thresholds are a function of $C_{B}$. Furthermore, as $C_{B}^{E}\left(q ; \sigma_{\sigma_{B}}\right)$ and $C_{B}^{R}\left(q ; \sigma_{\sigma_{B}}\right)$ are strictly increasing and continuous for $q \neq A_{R}$ and $q \in\left\{A_{R}, A_{E}\right\}$ respectively (c.f. Lemma 2), the

${ }^{31}$ In the model, $\kappa_{A}=\kappa_{B}=\kappa$; however, this result works in the more general case where colleges differ in their capacity. 
thresholds are increasing and continuous in $C_{B}$.

To see the dependence of $C_{B}$ on $\kappa_{B}$, consider the general version of equation 12 -college B's capacity constraint-i.e.,

$$
\begin{array}{r}
\int_{\left[\underline{B}_{E}\left(C_{B}\right), A_{R}\right) \cup\left[\bar{B}_{E}\left(C_{B}\right), 1\right]} S_{B}^{E}\left(q ; \Sigma_{\sigma_{B}} h\left(q \mid \mathcal{A}_{B}^{E}\left(\Sigma_{\sigma_{B}}\right)\right) d q+\right. \\
\int_{\left[\underline{B}_{R}\left(C_{B}\right), A_{E}\right) \cup\left[\hat{B}_{R}\left(C_{B}\right), A_{R}\right) \cup\left[\bar{B}_{R}\left(C_{B}\right), 1\right]} S_{B}^{R}\left(q ; \Sigma_{\sigma_{B}}\right) h\left(q \mid \mathcal{A}_{B}^{R}\left(\Sigma_{\sigma_{B}}\right)\right) d q=\kappa_{B} .
\end{array}
$$

The facts that the thresholds are increasing in $C_{B}$ and that $S_{B}^{p}\left(q ; \Sigma_{\sigma_{B}}\right) \geq 0$ imply, through equation 14 , that $C_{B}$ is decreasing in $\kappa_{B}$. Furthermore, note that if $\kappa_{B} \rightarrow 1-\kappa_{A}$, i.e. college B has capacity to admit all the students that college A will not enroll, $C_{B} \rightarrow 0$-every remaining student is admitted-whereas if $\kappa_{B} \rightarrow 0, C_{B} \rightarrow \sup _{q, p} C_{B}^{p}\left(q ; \Sigma_{\sigma_{B}}\right)$-no capacity means rejecting every applicant. Finally, as the thresholds are continuous in $C_{B}$ and the integral is continuous in the limits of integration, $C_{B}$ is continuous in $\kappa_{B}$.

Consider the case in which justified envy arises during the early process (the case for the regular process is analogous). A necessary condition for this to happen is that $\underline{C}=C_{B}^{E}\left(A_{R}^{-} ; \Sigma_{\sigma_{B}}\right)>$ $C_{B}^{E}\left(A_{R} ; \Sigma_{\sigma_{B}}\right)$ and $C_{B}^{E}\left(A_{R} ; \Sigma_{\sigma_{B}}\right)<C_{B}\left(\kappa_{B}\right)$ (c.f. Lemma 4). Due to the properties of $C_{B}\left(\kappa_{B}\right)$, it is possible to find $\kappa^{*}<1-\kappa_{A}$ such that if $\kappa_{B}^{\prime} \geq \kappa^{*}, C_{B}^{E}\left(A_{R} ; \Sigma_{\sigma_{B}}\right)>C_{B}\left(\kappa_{B}^{\prime}\right)$, and justified envy disappears. In this case, college B's optimal policy is a quality-thresholds one.

Now we prove the case of $\alpha_{B}$. Consider the early admissions process, for instance, when justified envy arises. Notice that $C_{B}^{E}\left(q ; \Sigma_{\sigma_{B}}, \alpha_{B}\right)$ is a strictly decreasing, continuous function of $\alpha_{B}$, for any $q$, with the property that $\left[\bar{C}-C_{B}^{E}\left(A_{R} ; \Sigma_{\sigma_{B}}, \alpha_{B}\right)\right] \rightarrow 0$ as $\alpha_{B} \rightarrow 0$. Thus, it is possible to find $\alpha_{E}^{*}$ such that if $\alpha_{B}<\alpha_{E}^{*}, C_{B}^{E}\left(A_{R} ; \Sigma_{\sigma_{B}}\right)>C_{B}$ and justified envy disappears. In this case, college B's admission policy becomes a quality-thresholds one too.

Lemma 6. If $\rho_{A} \geq \rho_{B}$, then

and

$$
\mathbb{P}\left(x_{B} \geq x_{A} \mid n=\bar{n}\right) \equiv 1-P\left(\rho_{A}, \rho_{B}\right)=\frac{\rho_{B}}{2 \rho_{A}},
$$

$$
\mathbb{E}\left(x_{B} \mid x_{B} \geq x_{A}, n=\bar{n}\right)=\rho_{B} \frac{4}{3} \bar{n}
$$

Proof of Lemma 6. Let $\frac{\rho_{A}}{\rho_{B}} \equiv \theta \geq 1$. Note that the event $x_{B} \geq x_{A}$, conditional on $n=\bar{n}$, is equivalent to the event $\left\{\hat{n}_{B} \geq \theta \hat{n}_{A}\right\}$. Also, conditional on $n=\bar{n}$, by assumption $\hat{n}_{A}$ and $\hat{n}_{B}$ are distributed uniformly on $[0,2 \bar{n}]$ and are independent $\left(\hat{n}_{j} \sim F\right.$ with density $\left.f\right)$. Finally, note that the value of $\theta$ restricts not only $\hat{n}_{B}$, but also $\hat{n}_{A}$, in terms of the integration region. Formally, in the $\mathbb{R}^{2}\left(\hat{n}_{B}, \hat{n}_{A}\right)$-plane, when $\theta \geq 1$, the integration region is the triangle with vertices in $\{(0,0),(2 \bar{n}, 0),(2 \bar{n}, 2 \bar{n} / \theta)\}$. 
With these observations, we have that

$$
\begin{gathered}
\mathbb{P}\left(x_{B} \geq x_{A} \mid n=\bar{n}\right)=\mathbb{P}\left(\hat{n}_{B} \geq \theta \hat{n}_{A} \mid n=\bar{n}\right)=\int_{0}^{2 \bar{n}} \mathbb{P}\left(\hat{n}_{A} \leq \frac{1}{\theta} \tilde{n} \mid n=\bar{n}, \hat{n}_{B}=\tilde{n}\right) f(\tilde{n} \mid n=\bar{n}) d \tilde{n}= \\
\frac{1}{\theta(2 \bar{n})^{2}} \int_{0}^{2 \bar{n}} \tilde{n} d \tilde{n}=\frac{1}{2 \theta},
\end{gathered}
$$

which proves the first part. Letting $\mathbb{I}(\Theta)$ be the indicator function of the event $\Theta$, we compute

$$
\begin{aligned}
\mathbb{E}\left(x_{B} \mathbb{I}\left(\hat{n}_{B} \geq \theta \hat{n}_{A}\right) \mid n=\bar{n}\right)= & \rho_{B} \mathbb{E}\left(\hat{n}_{B} \mathbb{I}\left(\hat{n}_{B} \geq \theta \hat{n}_{A}\right) \mid n=\bar{n}\right)=\rho_{B} \int_{0}^{2 \bar{n}} \tilde{n}_{B} \int_{0}^{\tilde{n}_{B} / \theta} f\left(\tilde{n}_{A}\right) f\left(\tilde{n}_{B}\right) d \tilde{n}_{A} d \tilde{n}_{B}= \\
& \frac{\rho_{B}}{(2 \bar{n})^{2}} \int_{0}^{2 \bar{n}} \tilde{n}_{B} \int_{0}^{\tilde{n}_{B} / \theta} d \tilde{n}_{A} d \tilde{n}_{B}=\frac{\rho_{B} 2 \bar{n}}{3 \theta}
\end{aligned}
$$

and thus, we conclude that

$$
\mathbb{E}\left(x_{B} \mid x_{B} \geq x_{A}, n=\bar{n}\right)=\frac{\mathbb{E}\left(x_{B} \mathbb{I}\left(\hat{n}_{B} \geq \theta \hat{n}_{A}\right) \mid n=\bar{n}\right)}{\mathbb{P}\left(\hat{n}_{B} \geq \theta \hat{n}_{A} \mid n=\bar{n}\right)}=\rho_{B} \frac{4}{3} \bar{n},
$$

which proves the second part.

\section{A.4 Theorems 2 to 4}

Proof of Theorem 2. Call the outcome of Theorem 1 the "early admissions outcome" or EAO. Consider the scenario where, all else equal, colleges are only allowed to use regular admissions and students apply accordingly. Proposition 1 implies that a unique subgame EA equilibrium exists, which we call the "regular-admissions-only outcome" or RAO.

Part (i). College B is strictly better off under the EAO, as shown in Proposition 5. College A is strictly worse off under the EAO because it is on the opposite side of the exchange of students that makes college B strictly better off.

Parts (ii) and (iii). To show the result for students, let $A_{E}, B_{E}$ and $B_{R}$ be colleges' EAO admission thresholds (recall that $B_{E}<\min \left\{B_{R}, A_{E}\right\}$ ), let $\hat{A}$ and $\hat{B}$ be college B's admission threshold in the RAO, and let $Y$ be the high-income indifferent student in the EAO. From the proof of Proposition 1, it follows that the enrollment pattern of the RAO is the following: every student with $q \in[\hat{B}, \hat{A})$ enrolls at college B. Low-income students with $q \geq \hat{A}$ are admitted to both colleges, a proportion $\frac{\rho_{B}}{2 \rho_{A}}$ (c.f. Lemma 6) enrolls at college B, and the rest at college A. High-income students with $q \geq \hat{A}$ are admitted to both colleges, the ones with $y<0$ enroll at college $\mathrm{B}$, and the rest at college A.

Likewise, from Proposition 2, the enrollment pattern of the EAO is the following: every high-income student with $(q, y) \in\left[B_{E}, 1\right] \times[\underline{y}, Y]$ enrolls early at college $\mathrm{B}$, whereas every high-income one with $(q, y) \in\left[A_{E}, 1\right] \times(Y, \bar{y}]$ enrolls early at college A. Low-income students with 
$q \geq \max \left\{B_{R}, A_{E}\right\}$ are admitted early to college $\mathrm{A}$ and regular to college $\mathrm{B}$, a proportion $\frac{\rho_{B}}{2 \rho_{A}}$ enrolls at college $\mathrm{B}$, and the rest at college $\mathrm{A}$. The low-income students with $q \in\left[A_{E}, \max \left\{B_{R}, A_{E}\right\}\right)$ enroll at college A. Finally, among students with $q \in\left[\min \left\{B_{R}, A_{E}\right\}, A_{E}\right)$, the low-income ones and the high-income ones with $y>\bar{Y}$ enroll at college B.

It is important to note the following: (i) $B_{E}<\hat{B}$, which is true because, in the EAO, low-income and high-income students with $y>Y$ apply early to college A, the ones with $q<A_{E}$ are rejected, and the ones with $q \in\left[B_{E}, \min \left\{A_{E}, B_{R}\right\}\right)$ are rejected as well from college B's early process. Therefore, if $B_{E} \geq \hat{B}$, college B would not be able to exhaust capacity in the EAO. And, (ii) the students of low-income and high-income with $y>Y$, for whom $q \in\left[B_{E}, \min \left\{A_{E}, B_{R}\right\}\right.$ ), enroll nowhere under the EAO, but enroll at college $\mathrm{B}$ under the RAO; this is true because, among said students, the ones with $q \in\left[\hat{B}, \min \left\{A_{E}, B_{R}\right\}\right)$ are rejected from college $\mathrm{B}$ in the EAO, but admitted in the RAO.

Consider the comparison of the EAO versus the RAO for high-income students. Although all them benefit from enrolling early instead of regular, their overall ex-ante welfare mainly depends on the comparison of the three groups for whom enrollment is different: the better-sorted, the downgraded and the expanded-access. The better-sorted and expanded-access students are ex post strictly better off, whereas the downgraded ones are ex post strictly worse-off.

The better-sorted ones are those with $(q, y) \in\left[B_{E}, \hat{B}\right] \times[\underline{Y}, 0]$ - which enroll at college $\mathrm{B}$, their favorite, instead of nowhere-and the ones with $(q, y) \in\left[A_{E}, \hat{A}\right] \times[Y, \bar{y}]$ - which enroll at college A, their favorite, instead of at college B. The downgraded ones are those with $(q, y) \in[\hat{A}, 1] \times[0, Y]$ - which enroll at college $\mathrm{B}$, their least favorite, instead of at college $\mathrm{A}$-and the ones with $(q, y) \in\left[\hat{B}, \min \left\{B_{R}, A_{E}\right\}\right] \times[Y, \bar{y}]$ when $B_{R}>\hat{B}$-which enroll nowhere, instead of at college B. The expanded-access ones are the ones with $(q, y) \in\left[B_{R}, \hat{B}\right] \times[Y, \bar{y}]$ when $B_{R}<\hat{B}$ - which enroll at college $\mathrm{B}$ instead of nowhere. Indeed, it is easy to see that the better-sorted group drives the aggregate ex-ante welfare of high-income students because, towards extreme tastes, their preference is very strong, i.e. $\lim _{y \rightarrow \bar{y}} v_{h}(A, y)=\lim _{y \rightarrow \underline{y}} v_{h}(B, y)=\infty$. This shows that high-income students are ex ante strictly better-off.

Finally, consider the comparison of the EAO versus the RAO for low-income students. If $B_{R}<A_{E}$, the ones with $q \in\left[A_{E}, \hat{A}\right]$ improve from enrolling at college $\mathrm{B}$ with one offer, to enrolling at either college $\mathrm{A}$ or $\mathrm{B}$, with the best of two offers. Formally, as $\bar{v}\left(\rho_{B}\right)>\frac{\bar{v}_{A}\left(\rho_{B}\right)}{\delta G^{*}}-$ so that low-income students wait for a second offer - these students have an improvement in utility of $\bar{v}\left(\rho_{B}\right)-\underline{v}_{B}\left(\rho_{B}\right)$. The low-income students with $q \in\left[\hat{B}, \max \left\{\hat{B}, B_{R}\right\}\right)$ however, miss college B's admission by applying early to college A. Their loss is $\underline{v}_{B}$. The rest of the low-income students do 
not affect their enrollment. As a result, it is possible to find $\bar{V}$, such that if $\bar{v}\left(\rho_{B}\right)>\bar{V}$, we have

$$
\begin{gathered}
{\left[G_{q}(\hat{A} \mid n=\bar{n}, y)-G_{q}\left(A_{E} \mid n=\bar{n}, y\right)\right]\left(\bar{v}\left(\rho_{B}\right)-\underline{v}_{B}\left(\rho_{B}\right)\right)>} \\
{\left[G_{q}\left(\max \left\{\hat{B}, B_{R}\right\} \mid n=\bar{n}, y\right)-G_{q}(\hat{B} \mid n=\bar{n}, y)\right] \underline{v}_{B}\left(\rho_{B}\right),}
\end{gathered}
$$

for every $y$. Note that if $\hat{B}>B_{R}$, the above expression is trivially true for any $\bar{V}$.

Proof of Theorem 3. For college A, as in Theorem 1, it is still optimal to offer $1=\rho_{A} \geq \rho_{B}$. Lemma 6 extends to an arbitrary $n$, so $\mathbb{P}\left(x_{B} \geq x_{A} \mid n\right)=\frac{1}{2} \rho_{B}$, this is, college A makes a better financial aid offer than college B more than half of the times. Colleges, again, find it optimal to use quality-threshold admission policies. We focus on the arguments from Theorem 1 that are modified when general preferences are considered.

-Early Admissions with $E_{B}=E D$ and $E_{A}=R E A$. From an analogous argument to the one in the proof of Proposition 2, college A must be more selective than college B-it has a better financial aid policy and it is more popular among students—so $B_{E}<B_{R}<1$ and $B_{E}<A_{E} \leq A_{R}<1$. Furthermore, $A_{E} \leq A_{R}$ gives students an incentive to apply early. However, to identify the optimal early application, now, both low- and high-income students face a trade-off between a better admission chance at college B and having to enroll without comparing financial aid. Furthermore, the students that prefer college A face the additional trade-off of having to enroll at their least preferred college.

Formally, if a $\bar{v}^{\tau}\left(\rho_{B}, y\right)=\mathbb{E}\left(\max \left\{v^{\tau}\left(A, x_{a}, y\right), v_{\tau}\left(B, x_{b}, y\right)\right\} \mid \rho_{A}=1, \rho_{B}, n, y\right)$ and $\underline{v}_{j}^{\tau}\left(\rho_{B}, y\right)=$ $\mathbb{E}\left(v_{\tau}\left(j, x_{j}, y\right) \mid \rho_{A}=1, \rho_{B}, n, y\right)$, a $\tau \in\{l, h\}$-type of income student applies early to college B if and only if:

$$
\begin{array}{r}
\left(1-G_{q}\left(B_{E} \mid y, n\right)\right) \underline{v}_{B}^{\tau}\left(\rho_{B}, y\right)> \\
\left(1-G_{q}\left(\max \left\{B_{R}, A_{E}\right\} \mid y, n\right)\right) \delta \bar{v}^{\tau}\left(\rho_{B}, y\right)+ \\
\left(G_{q}\left(\max \left\{B_{R}, A_{E}\right\} \mid y, n\right)-G_{q}\left(B_{R} \mid y, n\right)\right) \delta \underline{v}_{B}^{\tau}\left(\rho_{B}, y\right)+ \\
\left(G_{q}\left(\max \left\{B_{R}, A_{E}\right\} \mid y, n\right)-G_{q}\left(A_{E} \mid y, n\right)\right) \delta \underline{v}_{A}^{\tau}\left(\rho_{B}, y\right) .
\end{array}
$$

The assumption $\delta \bar{v}^{h}\left(\rho_{B}\right)<\underline{v}_{B}^{h}\left(\rho_{B}\right)$ for any $\rho_{B}$ implies that the high-income student with $y=0$ applies early to college $\mathrm{B}$. To see it, notice that as $\bar{v}^{h}\left(\rho_{B}, y\right) \geq \max \left\{\underline{v}_{B}^{h}\left(\rho_{B}, y\right), \underline{v}_{A}^{h}\left(\rho_{B}, y\right)\right\}$, Equation 15 is implied by

$$
\left(1-G_{q}\left(B_{E} \mid 0, n\right)\right) \underline{v}_{B}^{\tau}\left(\rho_{B}\right)>\left(1-G_{q}\left(\min \left\{B_{R}, A_{E}\right\} \mid 0, n\right)\right) \delta \bar{v}^{\tau}\left(\rho_{B}\right),
$$

which is implied by $\delta \bar{v}^{h}\left(\rho_{B}\right)<\underline{v}_{B}^{h}\left(\rho_{B}\right)$, given that $B_{E}<\leq \min \left\{B_{R}, A_{E}\right\}$. From the properties of $v_{h}$, it follows that there exists $Y_{h}>0$ such that if $y>Y_{h}$, high-income students apply early to college A, and to college B otherwise. 
The assumption that $\frac{1}{\delta G^{*}} \underline{v}_{A}^{l}\left(\rho_{B}\right)<\bar{v}^{l}\left(\rho_{B}\right)$ for any $\rho$, along with the observation that either $\left(G_{q}\left(\max \left\{B_{R}, A_{E}\right\} \mid y, n\right)-G_{q}\left(B_{R} \mid y, n\right)\right)=0$ or $\left(G_{q}\left(\max \left\{B_{R}, A_{E}\right\} \mid y, n\right)-G_{q}\left(A_{E} \mid y, n\right)\right)=0$, implies that the low-income student with $y=0$ finds it optimal to apply early to college A. The properties of $v_{l}$, analogously to the proof for high-income students in Proposition 2, implies that there exists $Y_{l}<0$ such that low-income students apply early to college A if $y>Y_{l}$, and to college $B$ otherwise. This leads to (iv') of Theorem 3, instead of to (iv) of Theorem 1.

The rest of the argument proceeds as in the proof of Theorem 1: the fact that $Y_{h}>0$ and $Y_{l}<0$ implies that $X_{B}^{E}\left(q ; \Sigma_{\sigma_{B}}\right)<X_{B}^{R}\left(q ; \Sigma_{\sigma_{B}}\right)$, which leads to consistency with $E_{B}<E_{R}$ via Equation 8. A self-map can be established, and it has a unique fixed point, which is a subgame EA equilibrium outcome. Finally, college A offers REA because ED makes it lose high-quality students $\left(Y_{h}\right.$ and $Y_{l}$ increase). Unlike the argument in Theorem 1, however, college A offers REA instead of no early admissions because it reduces the number of students it loses to college B via ED: the fact that $A_{E} \leq A_{R}$ implies that the student with $y=Y_{\tau}$ prefers to apply to college B if college A does not offer early admissions, as there is a benefit from applying early that is not present if college A offers only regular admissions.

-Regular admissions only. If no early admissions are present, students apply to both colleges regular. The students with $q \geq \max \left\{A_{R}, B_{R}\right\}$ are accepted to both colleges and get two financial aid offers; among these students, one of income type $\tau \in\{l, h\}$ and offers $\left\{x_{A}, x_{B}\right\}$ will enroll at college A only if $v_{\tau}\left(A, x_{A}, y\right)>v_{\tau}\left(B, x_{B}, y\right)$, and will enroll at college B otherwise. When $x_{A} \geq x_{B}$, $\frac{v_{\tau}\left(A, x_{A}, 0\right)}{v_{\tau}\left(B, x_{B}, 0\right)}>1$, so the student with taste $y=0$ prefers college A. Thus, the properties of $v_{\tau}$ imply that there exists $\bar{Y}_{\tau}\left(x_{A}, x_{B}\right) \leq 0$ such that if $y>\bar{Y}_{\tau}\left(x_{A}, x_{B}\right)$, then the student enrolls at college A. The properties of $v_{\tau}$ imply that $\bar{Y}_{\tau}\left(x_{A}, x_{B}\right)$ is decreasing in $x_{A}$ and increasing in $x_{B}$. In this case, $\mathbb{P}\left(y>\bar{Y}_{\tau}\left(x_{A}, x_{B}\right)\right)>\frac{1}{2}$ for $\tau \in\{l, h\}$, so college A enrolls more than half of the students with $x_{A} \geq x_{B}$.

The same reasoning implies that when $x_{B}>x_{A}$, there exists $\underline{Y}_{\tau}\left(x_{A}, x_{B}\right)>0$ such that college A enrolls the students with $y>\underline{Y}_{\tau}\left(x_{A}, x_{B}\right)$, which might or might not account for more than half of the students $\left(\mathbb{P}(y>0)>\frac{1}{2}\right)$. The properties of $v_{\tau}$ imply that $\underline{Y}_{\tau}\left(x_{A}, x_{B}\right)$ is decreasing in $x_{A}$ and increasing in $x_{B}$. However, as $\mathbb{P}\left(x_{B} \geq x_{A} \mid n\right)=\frac{1}{2} \rho_{B} \leq \frac{1}{2}$, we conclude that college A enrolls more than half of the students with $q \geq \max \left\{A_{R}, B_{R}\right\}$, and thus, college $\mathrm{B}$ must be less selective to exhaust capacity: $A_{R}>B_{R}$. Therefore, along with the previous enrollment patterns, college B enrolls all the students with $q \in\left[A_{R}, B_{R}\right]$.

As $u$ is strictly concave, Jensen's inequality applied to the college's aggregate utility from Equation 3 implies that college B strictly prefers to offer ED to no early admissions or REA, and 
the rest of the proof of Theorem 1 follows analogously to imply that there is a unique equilibrium outcome in which $E_{A}=R E A, E_{B}=E D$, and with the desired properties.

-Welfare. The welfare argument follows as in the proof of Theorem 1. For low-income students, welfare is dictated by an increase in the proportion of students that get two financial aid offers, given that their preferences for taste are not strong; for high-income students, welfare is dictated by a better sorting of students, given that their preferences for financial aid are not strong.

Proof of Theorem 4. We extend the arguments where $\alpha_{A}=0$ was used to simplify the analysis. Lemma 2. When $\alpha_{A}>0, C_{A}^{p}\left(q ; \Sigma_{\sigma_{A}}\right)=u(q)-\alpha_{A} X_{A}^{p}\left(q ; \Sigma_{\sigma_{A}}\right)$. The affiliation of $q$ and $-n$ implies that $X_{A}^{p}\left(q ; \Sigma_{\sigma_{A}}\right)$ is weakly decreasing in $q$, except for the students within a neighborhood of being admitted to college $\mathrm{B}$, case in which $X_{A}^{p}$ can be increasing if it enrolls low-income students that compare financial aid. As $u(q)$ is strictly increasing in $q$, however, there is $\bar{\alpha}>0$ such that if $\alpha_{A} \in[0, \bar{\alpha}]$, then $C_{A}^{p}\left(q ; \Sigma_{\sigma_{A}}\right)$ is strictly increasing in $q$, and the rest of the argument follows.

Lemma 3. The proportion of low-income students enrolled under a given financial aid policy $\rho_{A}$ for a given $\rho_{B}$ is, per Lemma $3,1-\frac{\rho_{B}}{2 \rho_{A}}$, which is increasing in $\rho_{A}$. Consider the thresholds $A_{R}^{\prime}>A_{R}$ and the financial aid policies $\rho_{A}^{\prime}>\rho_{A}$, such that college A exhausts capacity in both cases. If college A goes from $\rho_{A}$ to $\rho_{A}^{\prime}$, it gains $\frac{\rho_{B}}{2 \rho_{A}}-\frac{\rho_{B}}{2 \rho_{A}^{\prime}}$ of the low-income students with $q>A_{R}^{\prime}$ and it loses $G_{y}(0 \mid q, n)$ of the students with $q \in\left[A_{R}, A_{R}^{\prime}\right]$. College A strictly prefers the change if and only if

$$
\begin{array}{r}
\left(\frac{\rho_{B}}{2 \rho_{A}}-\frac{\rho_{B}}{2 \rho_{A}^{\prime}}\right) \int_{A_{R}^{\prime}}^{1}\left[u(q)-\alpha_{A} \mathbb{E}\left(x_{A} \mid x_{A} \geq x_{B}, n=\bar{n}\right)\right] h\left(q \mid \mathcal{A}_{j}^{p}\left(\Sigma_{\sigma_{j}}\right)\right) d q> \\
\int_{A_{R}}^{A_{R}^{\prime}}\left[u(q)-\alpha_{A} \mathbb{E}\left(x_{A} \mid n=\underline{n}\right)\right] G_{y}(0 \mid q, n) h\left(q \mid \mathcal{A}_{j}^{p}\left(\Sigma_{\sigma_{j}}\right)\right) d q .
\end{array}
$$

As college A exhausts capacity both at $A_{R}$ and $A_{R}^{\prime}$, the students swapped between the two scenarios account for the same capacity. From an analysis as the one in the proof of Lemma 1, it follows that if $\alpha_{A}=0$, Equation 16 holds. This, along with the fact that the integrands of Equation 16 are continuous in $\alpha_{A}$ and that $\mathbb{E}\left(x_{A} \mid x_{A} \geq x_{B}, n=\bar{n}\right)>\mathbb{E}\left(x_{A} \mid n=\underline{n}\right)$, implies that there exists $\bar{\alpha}$ such that if $\alpha_{A} \in[0, \bar{\alpha}]$, then Equation 16 holds, and the rest of Lemma 2 follows.

Proposition 2, when $E_{B}=E D$ and $E_{A}=E D$. If $\alpha_{A}>0$, an analogous of Equation 8 for college A implies that $A_{E}<A_{R}$. However, $\bar{v}\left(\rho_{B}\right)>\frac{\bar{v}_{A}\left(\rho_{B}\right)}{\delta G^{*}}$ implies that low-income students do not apply early anywhere. The fact that $B_{E}<A_{E}$ still implies that there is $Y>0$ such that high-income students with $y>Y$ apply early to college A, and to college B otherwise, and the rest of the argument follows through.

Welfare. The welfare arguments for students are unaffected by $\alpha_{A}>0$. 


\section{B Stylized Facts: Summary Statistics}

Table 1: Stylized Facts About the Top 25 Selective Private Colleges

\begin{tabular}{|c|c|c|}
\hline & REA & ED \\
\hline Colleges offering early admissions & 4 & 17 \\
\hline Colleges offering need-blind admissions & $100 \%$ & $82 \%$ \\
\hline \multicolumn{3}{|l|}{ College characteristics: } \\
\hline USN General Ranking & 3.3 & 18.2 \\
\hline Revealed Preference Ranking & 3.75 & 28.6 \\
\hline USN Financial Resources Ranking & 5.75 & 21.5 \\
\hline Endowment per student after expenditures & $\$ 1,967,625$ & $\$ 374,281$ \\
\hline Cost of attendance & $\$ 66,719$ & $\$ 69,573$ \\
\hline \multicolumn{3}{|l|}{ Admissions: } \\
\hline Applications & 36,878 & 32,729 \\
\hline$\%$ of early applications & $16 \%$ & $9 \%$ \\
\hline Enrolled students (freshmen) & 1,411 & 2,010 \\
\hline$\%$ of class enrolled from early applicants & $54 \%$ & $43 \%$ \\
\hline Ratio of total applications to class size & 27.3 & 17.3 \\
\hline Yield rate & $67 \%$ & $44 \%$ \\
\hline Admission rate (overall) & $5.8 \%$ & $15.6 \%$ \\
\hline \multicolumn{3}{|l|}{ Applicants' characteristics: } \\
\hline SAT of incoming class (out of 1,600 ) & 1,485 & 1,451 \\
\hline High school GPA of incoming class (out of 4) & 3.9 & 3.8 \\
\hline Freshmen in the top $10 \%$ of high school class & $93 \%$ & $85 \%$ \\
\hline \multicolumn{3}{|l|}{ Among need-blind colleges only } \\
\hline \multicolumn{3}{|l|}{ Need-based financial aid: } \\
\hline Full-need coverage for US citizens & $100 \%$ & $65 \%$ \\
\hline Full-need coverage for internationals & $75 \%$ & $0 \%$ \\
\hline \multicolumn{3}{|l|}{ Among colleges that cover full need } \\
\hline Ratio of non-repayable to repayable aid & 25 & 12 \\
\hline Average paid by a student with income $<\$ 48,000$ & $\$ 2,160$ & $\$ 7,335$ \\
\hline Average paid by a student with income $>\$ 110,000$ & $\$ 37,184$ & $\$ 43,334$ \\
\hline
\end{tabular}

Note: No significance levels are reported as the sample is small.

Source: IPEDS, CDS, CB, USN, CWP, and Avery et al. (2012). 


\section{Example (for Online Publication).}

Regular Admissions. If admitted, college A makes a better offer to a low-income student than college B in 3/4 of the times. Also, 2/3 of the high-income students prefer college A over college B. As a result, to exhaust capacity by admitting the students with $q \geq A_{R}$, college A sets $A_{R} \approx 0.42$ that solves the capacity constraint problem

$$
\left(1-A_{R}\right) \cdot\left[\frac{1}{4} \cdot \frac{3}{4}+\frac{3}{4} \cdot \frac{2}{3}\right]=\frac{1}{3} .
$$

If admitted, among students with $q \geq A_{R}$, college B enrolls the remaining students, as well as every student with $q<A_{R}$. Then, to exhaust capacity, college B sets $B_{R}=\frac{1}{3}$ that solves the capacity constraint problem

$$
\left(1-A_{R}\right) \cdot\left[\frac{1}{4} \cdot \frac{1}{4}+\frac{3}{4} \cdot \frac{1}{3}\right]+\left(A_{R}-B_{R}\right)=\frac{1}{3} .
$$

This scenario is consistent with no justified envy because $C_{B}=C_{B}^{R}\left(B_{R} ; \Sigma_{\sigma_{B}}\right) \approx 0.32<$ $C_{B}^{R}\left(A_{R} ; \Sigma_{\sigma_{B}}\right) \approx 0.49$.

Regular Admissions. If admitted, the students with $y>Y$ enroll at college A. Likewise, the low-income students with $q \geq \max \left\{A_{E}, B_{R}\right\}$ are admitted to both colleges and college A makes the best offer $3 / 4$ of the times. Finally, the low-income students with $q \in\left[A_{E}, \max \left\{A_{E}, B_{R}\right\}\right]$ are only admitted to college $\mathrm{A}$ and enroll. As a result, college A exhausts capacity at $A_{E}$ that solves

$$
\frac{3}{4} \cdot\left(1-A_{E}\right) \cdot\left(\frac{2}{3}-Y\right)+\frac{1}{4} \cdot\left[\frac{3}{4} \cdot\left(1-\max \left\{A_{E}, B_{R}\right\}\right)+\left(\max \left\{A_{E}, B_{R}\right\}-A_{E}\right)\right]=\frac{1}{3} \text {. }
$$

College B's ED binding commitment enrolls the high-income students with $y<Y$ that are admitted. College $\mathrm{B}$ also makes a better offer than college $\mathrm{A}$ to $1 / 4$ of the low-income students that are admitted regular. Finally, college B enrolls all the admitted regular applicants that were rejected by college A. As a result, college B exhausts capacity at $B_{E}$ and $B_{R}$ that solve

$$
\frac{3}{4} \cdot\left(1-B_{E}\right)+\frac{1}{4} \cdot \frac{1}{4} \cdot\left(1-\max \left\{A_{E}, B_{R}\right\}\right)+\left(A_{E}-\min \left\{B_{R}, A_{E}\right\}\right) \cdot\left(\frac{1}{4}+\frac{3}{4} \cdot\left(\frac{2}{3}-Y\right)\right)=\frac{1}{3} .
$$

These two conditions, along with equations 2 and 1 in the main text define a four equation, four unknowns system whose solution is the subgame EA equilibrium. As only high-income students apply early to college B and $\underline{n}=0, X_{B}^{E}\left(B_{E} ; \Sigma_{\sigma_{B}}\right)=0$. Regular, however, apply the low-income students and the high-income with $y>Y$ that were rejected to college A early. Therefore $X_{B}^{R}\left(B_{R} ; \Sigma_{\sigma_{B}}\right)=\frac{1 / 4}{1 / 4+3 / 4 \cdot(2 / 3-Y)} \cdot \frac{1}{2} \cdot 1$. The solution to this system is $A_{E} \approx 0.4, B_{E} \approx 0.32, B_{R} \approx 0.34$ and $Y \approx 0.18$. This scenario is consistent with no justified envy because $C_{B}=C_{B}^{R}\left(B_{R} ; \Sigma_{\sigma_{B}}\right) \approx$ $0.32<C_{B}^{R}\left(A_{R} ; \Sigma_{\sigma_{B}}\right) \approx 0.33$. 


\section{Data Sources, Variables and Anecdotal Evidence (for Online Publication)}

My database, my code and the documentation of the variables can be found in the following github repository: https://github.com/antonzm99/EarlyAdmissions

\section{D.1 Data Sources}

My data from the IPEDS, CDS and US and News World Report, is for the 2018 academic year. For the former two cases, I textually transcribe the description that the source provide.

Integrated Post Secondary Data System (IPEDS): It is a survey managed by the National Center for Education Statistics. IPEDS data are submitted at the aggregate-level from postsecondary institutions and do not have student-level information. Institutions submit data through 12 interrelated survey components about 6 general higher education topics in 3 collection cycles. Data on 12-month enrollment, academic libraries, admissions, completions, fall enrollment, college finance, graduation rates, human resources, institutional characteristics, outcome measures, and student financial aid are reported.

Common Data Set (CDS): The CDS initiative is a collaborative effort among data providers in the higher education community and publishers as represented by the College Board, Peterson's, and U.S. News \& World Report. The combined goal of this collaboration is to improve the quality and accuracy of information provided to all involved in a student's transition into higher education, as well as to reduce the reporting burden on data providers. The CDS is a set of standards and definitions of data items rather than a survey instrument or set of data represented in a database. Each of the higher education surveys conducted by the participating publishers incorporates items from the CDS as well as unique items proprietary to each publisher. Consequently, the publishers' surveys differ in that they utilize varying numbers of items from the CDS.

Avery et al. (2009): It is a book about early admissions that summarizes the evidence of the “Colleges Admission Project," which surveys more than 80,000 high school and college students. This source also gathers historic and anecdotal evidence on the origins and development of early admissions. Part of the evidence it compiles includes testimonials and interviews with college officers, counselors, students, parents and high school authorities.

Avery and Levin (2010): This article offers a more formal analysis on the evidence from the "Colleges Admission Project." than Avery et al. (2009). Its focus, however, is to explain how early 
admissions help colleges improve the fit match with their admitted students.

US News and World Report: It is one of the leading independent institutions specialized in college admissions. On a yearly basis, it gathers information from other sources and constructs useful indicators, such as colleges overall and financial rankings.

The Chronicle of Higher Education: It is a news source founded in 1966. Nowadays, it has the US's largest newsroom dedicated to covering colleges and universities. It is the unrivaled leader in higher education journalism, providing real-time news and deep insights about the market.

\section{D.2 Sample}

The sample of colleges used in my analysis was selected in the following way. First, I ranked colleges according to the USN ranking. Second, I selected the top 25 private-ones that were deemed as "most selective" by USN. Third, I discarded the colleges not offering early admissions. Among the remaining colleges, I determined the ones that were offering only ED or REA. Finally, I discarded the colleges offering other formats or more than one program. The colleges in each group are the following:

REA colleges: Harvard, Stanford, Princeton and Yale.

ED colleges: Boston University, Brandeis University, Brown, Carnegie Mellon, Columbia, Cornell, Dartmouth, Duke, Emory, Johns Hopkins, NYU, Northwestern, Rice, Tufts, UPenn, Vanderbilt, and Washington University in St. Louis.

No early admissions: Boston College, and University of Southern California (USC).

Different formats or more than one program: Chicago, the M.I.T., CalTech, and Georgetown.

\section{D.3 Variables}

Most of the variables were used as obtained from the sources. I make further clarification about some of them. The US News and World Report Ranking and the US News and World Report Financial Resources Rankings are two variables constructed and reported by the institution itself. The former is based on a general index that considers college traits of interest for students: graduation and retention rates, social mobility, graduate performance, academic reputation, faculty resources, selectivity, financial resources, and alumni giving rate. It does not consider selectivity or yield rates, however. The latter is based on an index about the financial attractiveness of the colleges. It considers average spending per student on instruction, research, student services and relational expenditures, without considering non-academic expenditures such as spending in sports or dorms. 
The full-time equivalent (FTE) student is a measurement equal to one student enrolled full-time for one academic year. Total FTE enrollment includes full-time plus the calculated equivalent of the part-time enrollment. Self-help aid is financial aid in the form of student loans, work-study positions and any other repayable methods, as opposed to scholarships and grants, that are gifted to students.

The main calculated variable was the proportion of early applicants. To construct it, I multiplied the proportion of the class enrolled through early admissions, by the class size, to obtain the number of applicants enrolled from early admissions. To compute the number of early applicants, I divided the number of applicants enrolled through early admissions, by the admission rate among such students. Finally, I found the proportion of early applicants by dividing the number of early applicants by the number of total applicants.

Meanwhile, I calculated the cost of attendance as the tuition plus the cost of room and boarding. Finally, the ratio of nonrepayable to repayable aid was calculated by dividing the amount of aid provided in scholarships and grants by the amount of aid provided in loans, work-study and other sources of repayable aid. 